\title{
FRACTIONAL KPZ EQUATIONS WITH CRITICAL GROWTH IN THE GRADIENT RESPECT TO HARDY POTENTIAL
}

\author{
BOUMEDIENE ABDELLAOUI, IRENEO PERAL, ANA PRIMO, FERNANDO SORIA
}

\begin{abstract}
In this work we study the existence of positive solution to the fractional quasilinear problem,$$
\left\{\begin{aligned}
(-\Delta)^{s} u & =\lambda \frac{u}{|x|^{2 s}}+|\nabla u|^{p}+\mu f & & \text { in } \Omega, \\
u & >0 & & \text { in } \Omega, \\
u & =0 & & \text { in }\left(\mathbb{R}^{N} \backslash \Omega\right),
\end{aligned}\right.
$$

where $\Omega$ is a $C^{1,1}$ bounded domain in $\mathbb{R}^{N}, N>2 s, \mu>0, \frac{1}{2}<s<1$, and $0<\lambda<\Lambda_{N, s}$ is defined in (3). We assume that $f$ is a non-negative function with additional hypotheses.

As we will see, there are deep differences with respect to the case $\lambda=0$. More precisely,

- If $\lambda>0$, there exists a critical exponent $p_{+}(\lambda, s)$ such that for $p>p_{+}(\lambda, s)$ there is no positive solution.

- Moreover, $p_{+}(\lambda, s)$ is optimal in the sense that, if $p<p_{+}(\lambda, s)$ there exists a positive solution for suitable data and $\mu$ sufficiently small.
\end{abstract}

To Shair Ahmad in his 85th birthday with our friendship and recognition.

\section{INTRODUCTION}

This work deals with the following problem:

$$
\left\{\begin{aligned}
(-\Delta)^{s} u & =\lambda \frac{u}{|x|^{2 s}}+|\nabla u|^{p}+\mu f & & \text { in } \Omega, \\
u & >0 & & \text { in } \Omega, \\
u & =0 & & \text { in }\left(\mathbb{R}^{N} \backslash \Omega\right),
\end{aligned}\right.
$$

where $0<\lambda<\Lambda_{N . s}$ defined in (3), $\mu>0, s \in\left(\frac{1}{2}, 1\right), 2 s<N, \Omega \subset \mathbb{R}^{N}$ is a bounded regular domain containing the origin and $f$ is a measurable non-negative function satisfying suitable hypotheses.

By $(-\Delta)^{s}$ we denote the fractional Laplacian of order $2 s$ introduced by M. Riesz in [29], that is,

$$
(-\Delta)^{s} u(x):=a_{N, s} \text { P.V. } \int_{\mathbb{R}^{N}} \frac{u(x)-u(y)}{|x-y|^{N+2 s}} d y, \quad s \in(0,1),
$$

where

$$
a_{N, s}=2^{2 s-1} \pi^{-\frac{N}{2}} \frac{\Gamma\left(\frac{N+2 s}{2}\right)}{|\Gamma(-s)|}
$$

Date: February 7, 2020.

Work partially supported by Project MTM2016-80474-P, MINECO, Spain.

The first author is also partially supported by DGRSDT, Algeria.

2010 Mathematics Subject Classification. 47G20, 35J75, 35J62, 35R09.

Keywords. Fractional elliptic equations, nonlinear term in the gradient, Hardy potential, stationary Kardar-ParisiZhang equations, Existence and Nonexistence results. 
is the normalizing constant that gives the Fourier multiplier identity

$$
\mathcal{F}\left((-\Delta)^{s} u\right)(\xi)=|\xi|^{2 s} \mathcal{F}(u)(\xi), \text { for } u \in \mathscr{S}\left(\mathbb{R}^{N}\right)
$$

See [19] for details.

For $\lambda=0$, in [6] (see also version [7]), the authors study natural conditions on $f$ in order to determine the existence of a positive solution to the problem (1) depending on the value of $p$. There are three cases: subcritical, $p<2 s$, critical $p=2 s$ and supercritical $p>2 s$.

For $\lambda>0$, the problems studied in this article are related to the following Hardy inequality, proved in [25] (see also [10, 19, 32, 33] and the monograph [28] for a detailed proof).

Theorem 1.1. (Fractional Hardy inequality). For all $u \in \mathcal{C}_{0}^{\infty}\left(\mathbb{R}^{N}\right)$ the following inequality holds,

$$
\int_{\mathbb{R}^{N}}|\xi|^{2 s}|\hat{u}|^{2} d \xi \geqslant \Lambda_{N, s} \int_{\mathbb{R}^{N}}|x|^{-2 s} u^{2} d x
$$

where

$$
\Lambda_{N, s}=2^{2 s} \frac{\Gamma^{2}\left(\frac{N+2 s}{4}\right)}{\Gamma^{2}\left(\frac{N-2 s}{4}\right)} .
$$

The constant $\Lambda_{N, s}$ is optimal and not attained.

Notice that, as it was stated in [19], the fractional Hardy's inequality plays an important role in the proof of the stability of relativistic matter in a very general setting.

It is clear that the criticality of the inequality is motivated by the homogeneity between the fractional Laplacian and the inverse $2 s$-potential. Moreover, letting $s \rightarrow 1$, then one can prove that

$$
\Lambda_{N, s} \rightarrow \Lambda_{N, 1}:=\left(\frac{N-2}{2}\right)^{2},
$$

the classical Hardy constant.

Notice that the optimal constant defined in (3) coincides for every bounded domain $\Omega$ containing the pole of the Hardy potential. That is, if $0 \in \Omega$, we can rewrite the Hardy inequality (2) as

$$
\frac{a_{N, s}}{2} \int_{Q} \frac{|u(x)-u(y)|^{2}}{|x-y|^{N+2 s}} d x d y \geqslant \Lambda_{N, s} \int_{\Omega} \frac{u^{2}}{|x|^{2 s}} d x, u \in H_{0}^{s}(\Omega) \text {. }
$$

The optimality of $\Lambda_{N, s}$ here follows by a scaling argument.

Related to problem (1), in the local case $s=1$ and for $0<\lambda<\Lambda_{N, 1}$ fixed, the authors in [5] identify a critical exponent $p_{+}(\lambda)$ such that for $p \geqslant p_{+}$, there exists no positive weak solution and for $1<p<p_{+}, \mu$ sufficiently small, and $f \leqslant \frac{1}{|x|^{2}}$, they prove the existence of a weak positive solution.

Problem (1) can be seen as the stationary Kardar-Parisi-Zhang problem with fractional diffusion and under the influence of the uncertainty principle given by the Hardy inequality. The classical model by Kardar-Parisi-Zhang was introduced in [23] with diffusion driven by the Laplacian. In the fractional setting see [21].

Our aim in this work is to analyze the case $s \in\left(\frac{1}{2}, 1\right)$ and $\lambda>0$. Notice that $s>\frac{1}{2}$ ensures the ellipticity of the problem. Our main result is the following one.

Theorem 1.2. Assume that $s \in\left(\frac{1}{2}, 1\right)$ and $0<\lambda<\Lambda_{N, s}$, then there exists a critical exponent $p_{+}(\lambda, s)>0$ such that if $p>p_{+}(\lambda, s)$ there is no positive solution to problem (1). Moreover, if $p<p_{+}(\lambda, s)$, problem (1) has a positive solution for suitable data and $\mu$ sufficiently small. 
The paper is organized as follows. In Section 2, we give the notion of solution that we are going to consider here. Moreover, we study the behavior of radial potential solutions of the homogenous problem in the whole space. Section 3 is devoted to the non existence of solutions. In that respect, we obtain two types of non existence results.

- On the one hand, we prove the existence of $p_{+}(\lambda, s)$ such that if $s \in\left(\frac{1}{2}, 1\right)$ and $p>p_{+}(\lambda, s)$, for all $\lambda>0$, the problem has no positive solution in a weak sense.

- On the other, we prove that for $s \in\left(\frac{1}{2}, 1\right)$, there exists $\mu^{*}>0$ such that if $\mu>\mu^{*}$, the problem has no positive solution for any $p$; that is, the positive source term must be small enough to ensure the existence of solutions.

Section 4 is devoted precisely to the existence of solutions. For $p<p_{+}(\lambda, s)$ and under additional hypotheses on the integrability of $f$, we are able to build a suitable supersolution and then by a monotonicity argument, to prove the existence of a minimal positive solution for all $\mu$. Moreover, for $p<\frac{N}{N-2 s+1}$, and for all $f \in L^{1}(\Omega)$ that satisfies a suitable integral condition near the origin, we prove the existence of $\mu^{*}$ such that for $\mu<\mu^{*}$, there exists a positive solution.

In the last section, we treat the case where the gradient term depends also on a zero order term. In this case under a suitable behavior of the zero order term at infinity, we are able to show the existence of a solution for all $p<2 s$, under suitable hypotheses on the data. It is worthy to point out that,in the local case, this last problem comes from the elliptic part of a porous medium equation, see [3].

\section{Preliminary Results}

Before starting the analysis of existence and non existence of positive solution, let us begin describing the precise sense in which solutions are defined. Consider the problem

$$
\left\{\begin{array}{rll}
(-\Delta)^{s} u & =\text { in } \Omega, \\
u & >0 & \text { in } \Omega, \\
u & =0 & \text { in } \mathbb{R}^{N} \backslash \Omega,
\end{array}\right.
$$

where $g \in L^{1}(\Omega)$.

Definition 2.1. We define the class of test functions

$$
\mathcal{T}(\Omega)=\left\{\phi \mid(-\Delta)^{s}(\phi)=\psi \text { in } \Omega, \quad \phi=0 \text { in } \mathbb{R}^{N} \backslash \Omega, \quad \psi \in \mathcal{C}_{0}^{\infty}(\Omega)\right\} .
$$

Notice that if $v \in \mathcal{T}(\Omega)$ then, using the results in [26], $v \in H_{0}^{s}(\Omega) \cap L^{\infty}(\Omega)$. Moreover, according to the regularity theory developed in [31], if $\Omega$ is smooth enough, there exists a constant $\beta>0$ (that depends only on the structural constants) such that $v \in \mathcal{C}^{\beta}(\Omega)$ (see also [22]).

Definition 2.2. We say that $u \in L^{1}(\Omega)$ is a weak solution to (5) if for $g \in L^{1}(\Omega)$ we have that

$$
\int_{\Omega} u \psi d x=\int_{\Omega} g \phi d x
$$

for any $\phi \in \mathcal{T}(\Omega)$ with $\psi \in \mathcal{C}_{0}^{\infty}(\Omega)$.

Recall also the definition of the truncation operator $T_{k}$,

$$
T_{k}(\sigma)=\max \{-k ; \min \{k, \sigma\}\} .
$$

From [26], [16] and [1] we have the next existence result. 
Theorem 2.3. Suppose that $g \in L^{1}(\Omega)$, then problem (5) has a unique weak solution u obtained as the limit of $\left\{u_{n}\right\}_{n \in \mathbb{N}}$, the sequence of unique solutions to the approximating problems

$$
\left\{\begin{aligned}
(-\Delta)^{s} u_{n} & =g_{n}(x) & & \text { in } \Omega \\
u_{n} & =0 & & \text { in } \mathbb{R}^{N} \backslash \Omega,
\end{aligned}\right.
$$

with $g_{n}=T_{n}(g)$. Moreover,

and

$$
\begin{gathered}
T_{k}\left(u_{n}\right) \rightarrow T_{k}(u) \text { strongly in } H_{0}^{s}(\Omega), \quad \forall k>0, \\
u \in L^{q}, \quad \forall q \in\left(1, \frac{N}{N-2 s}\right)
\end{gathered}
$$

In addition, if $s>\frac{1}{2}$, then $u \in W_{0}^{1, q}(\Omega)$ for all $q<\frac{N}{N-(2 s-1)}$ and $u_{n} \rightarrow u$ strongly in $W_{0}^{1, q}(\Omega)$.

Now, before dealing with the main problem (15), let us introduce the following definition.

Definition 2.4. Assume that $f \in L^{1}(\Omega)$ is a nonnegative function. We say that $u$ is a solution to problem (1) if $u \in W_{0}^{1, p}(\Omega), \frac{u}{|x|^{2 s}} \in L^{1}(\Omega)$ and, setting $g \equiv \lambda \frac{u}{|x|^{2 s}}+|\nabla u|^{p}+f$, then $u$ is a weak solution to problem (5) in the sense of Definition 2.2.

In order to study the behavior in a neighborhood of the origin of a nonnegative solution to problem (1), we need to analyze each radial potential positive solution in the whole space. More precisely, let us consider the homogeneous problem

$$
(-\Delta)^{s} u=\lambda \frac{u}{|x|^{2 s}} \text { in } \mathbb{R}^{N} \backslash\{0\}
$$

where $0<\lambda \leqslant \Lambda_{N, s}$. Then we have (see for instance [32, Theorem 4.1])

Lemma 2.5. Let $0<\lambda \leqslant \Lambda_{N, s}$. Then $v_{ \pm \alpha_{\lambda}}(x)=|x|^{-\frac{N-2 s}{2} \pm \alpha_{\lambda}}$ are solutions to problem (12), where $\alpha_{\lambda}$ is obtained by the identity

$$
\lambda=\lambda\left(\alpha_{\lambda}\right)=\lambda\left(-\alpha_{\lambda}\right)=\frac{2^{2 s} \Gamma\left(\frac{N+2 s+2 \alpha_{\lambda}}{4}\right) \Gamma\left(\frac{N+2 s-2 \alpha_{\lambda}}{4}\right)}{\Gamma\left(\frac{N-2 s+2 \alpha_{\lambda}}{4}\right) \Gamma\left(\frac{N-2 s-2 \alpha_{\lambda}}{4}\right)} .
$$

Remark 2.6. Notice that $\lambda(\alpha)=\lambda(-\alpha)=m_{\alpha_{\lambda}} m_{-\alpha_{\lambda}}$, with $m_{\alpha_{\lambda}}=2^{\alpha_{\lambda}+s} \frac{\Gamma\left(\frac{N+2 s+2 \alpha_{\lambda}}{4}\right)}{\Gamma\left(\frac{N-2 s-2 \alpha_{\lambda}}{4}\right)}$.

Lemma 2.7. The following equivalence holds true:

$$
0<\lambda\left(\alpha_{\lambda}\right)=\lambda\left(-\alpha_{\lambda}\right) \leqslant \Lambda_{N, s} \text { if and only if } 0 \leqslant \alpha_{\lambda}<\frac{N-2 s}{2} .
$$

For an elementary proof of this Lemma see $[4,19,25]$.

Remark 2.8. Denote

$$
\mu(\lambda)=\frac{N-2 s}{2}-\alpha_{\lambda} \text { and } \bar{\mu}(\lambda)=\frac{N-2 s}{2}+\alpha_{\lambda} .
$$

For $0<\lambda<\Lambda_{N, s}$, then $0<\mu<\frac{N-2 s}{2}<\bar{\mu}<(N-2 s)$. Since $N-2 \mu-2 s=2 \alpha_{\lambda}>0$ and $N-2 \bar{\mu}-2 s=-2 \alpha_{\lambda}<0$, then $(-\Delta)^{s / 2}\left(|x|^{-\mu}\right) \in L^{2}(\Omega)$, but $(-\Delta)^{s / 2}\left(|x|^{-\bar{\mu}}\right)$ does not. 
As a consequence we have the next comparison lemma.

Lemma 2.9. Assume that $u \in L_{l o c}^{1}\left(\mathbb{R}^{N}\right)$ is such that $u \geqslant 0$ in $\mathbb{R}^{N}$ with $(-\Delta)^{s} u \in L_{\text {loc }}^{1}(\Omega)$. Suppose that

then

$$
(-\Delta)^{s} u \geqslant \lambda \frac{u}{|x|^{2 s}} \text { in } \Omega, \quad 0<\lambda<\Lambda_{N, s},
$$

$$
u(x) \geqslant C|x|^{-\mu(\lambda)}=C|x|^{-\frac{N-2 s}{2}+\alpha_{\lambda}} \text { in } B_{r}(0) \subset \subset \Omega .
$$

See [4] for a detailed proof.

\section{NON EXISTENCE RESUlT}

We now consider the problem stated in the introduction

$$
\left\{\begin{aligned}
(-\Delta)^{s} u & =\lambda \frac{u}{|x|^{2 s}}+|\nabla u|^{p}+\mu f & & \text { in } \Omega, \\
u & >0 & & \text { in } \Omega, \\
u & =0 & & \text { in } \mathbb{R}^{N} \backslash \Omega,
\end{aligned}\right.
$$

where $\Omega \subset \mathbb{R}^{N}$ is a bounded regular domain containing the origin, $0<\lambda<\Lambda_{N, s}, \mu>0, s \in\left(\frac{1}{2}, 1\right)$, $p>1$ and $f$ is a non-negative function.

To establish the upper bound for $p$ we follow closely the arguments of [5], see also [14] for the potential case. We look for a radial solution to the problem (15).

$$
(-\Delta)^{s} w-\lambda \frac{w}{|x|^{2 s}}=|\nabla w|^{p} \text { in } \mathbb{R}^{N} .
$$

In particular, if we choose $w=A|x|^{\beta-\frac{N-2 s}{2}}$, with $A$ a positive constant, $0<\beta<\frac{N-2 s}{2}$, then (16) is equivalent to have

$$
A \gamma_{\beta}|x|^{-2 s-\frac{N-2 s}{2}+\beta}-\lambda A|x|^{\beta-2 s-\frac{N-2 s}{2}}=\frac{A\left|\beta-\frac{N-2 s}{2}\right|^{p}}{|x|^{\left(\frac{N-2 s}{2}-\beta+1\right) p}},
$$

where

$$
\gamma_{\beta}:=\gamma_{-\beta}:=\frac{2^{2 s} \Gamma\left(\frac{N+2 s+2 \beta}{4}\right) \Gamma\left(\frac{N+2 s-2 \beta}{4}\right)}{\Gamma\left(\frac{N-2 s-2 \beta}{4}\right) \Gamma\left(\frac{N-2 s+2 \beta}{4}\right)} .
$$

Hence, in order to have homogeneity we need

$$
p=\frac{\frac{N-2 s}{2}-\beta+2 s}{\frac{N-2 s}{2}-\beta+1},
$$

which means that $\beta=\frac{N-2 s}{2}+\frac{p}{p-1}-\frac{2 s}{p-1}$ and, then, the constants must satisfy the equation $\gamma_{\beta}-\lambda=$ $A^{p-1}\left|\beta-\frac{N-2 s}{2}\right|^{p}$.

Since $A>0$, we need $\gamma_{\beta}-\lambda>0$. Consider the map

$$
\begin{aligned}
\Upsilon:\left(-\frac{N-2 s}{2}, \frac{N-2 s}{2}\right) & \mapsto\left(0, \Lambda_{N, s}\right) \\
\beta & \mapsto \gamma_{\beta}
\end{aligned}
$$

then $\Upsilon$ is even and the restriction of $\Upsilon$ to the set $\left[0, \frac{N-2 s}{2}\right)$ is decreasing, see [15] and [19], so there exists a unique $\alpha_{\lambda} \in\left(0, \Lambda_{N, s}\right]$ such that $\gamma_{\alpha_{\lambda}}=\gamma_{-\alpha_{\lambda}}=\lambda$. 
Let $\beta_{0}=-\beta_{1}=\alpha_{\lambda}$, therefore, setting

$$
p_{+}(\lambda, s):=\frac{\frac{N-2 s}{2}-\beta_{0}+2 s}{\frac{N-2 s}{2}-\beta_{0}+1}=\frac{N+2 s-2 \alpha_{\lambda}}{N-2 s-2 \alpha_{\lambda}+2},
$$

and

$$
p_{-}(\lambda, s):=\frac{\frac{N-2 s}{2}-\beta_{1}+2 s}{\frac{N-2 s}{2}-\beta_{1}+1}=\frac{N+2 s+2 \alpha_{\lambda}}{N-2 s+2 \alpha_{\lambda}+2},
$$

it holds that $p_{-}(\lambda, s)<p_{+}(\lambda, s)$ and $\gamma_{\beta}-\lambda>0$ if and only if

$$
p_{-}(\lambda, s)<p<p_{+}(\lambda, s) \text {. }
$$

It is easy to check that $p_{+}(\lambda, s)$ and $p_{-}(\lambda, s)$ are respectively an increasing and a decreasing function in $\alpha_{\lambda}$ and, therefore, are respectively a decreasing and an increasing function in $\lambda$. Thus

$$
\frac{N}{N-2 s+1}<p_{-}(\lambda, s)<\frac{N+2 s}{N-2 s+2}<p_{+}(\lambda, s)<2 s, \text { for } 0<\lambda<\Lambda_{N, s}
$$

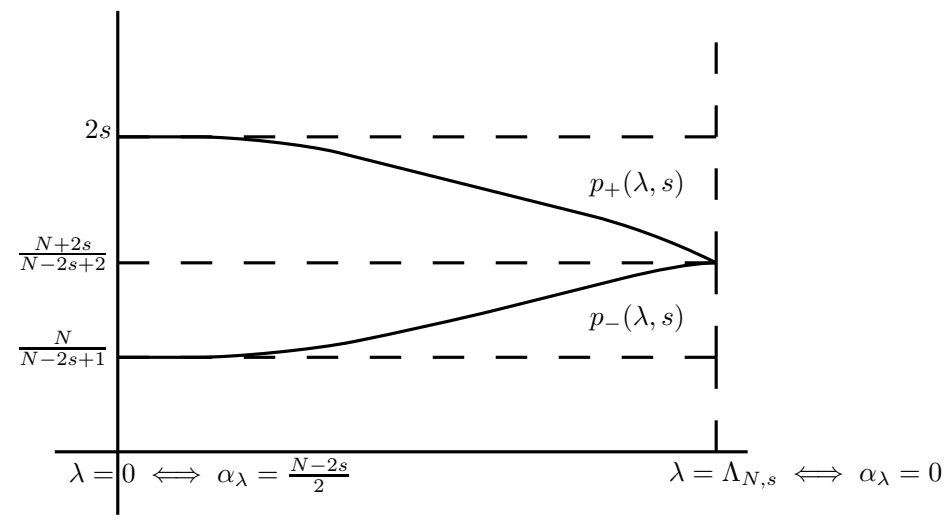

Recalling that $\mu(\lambda)=\frac{N-2 s}{2}-\alpha_{\lambda}, \bar{\mu}(\lambda)=\frac{N-2 s}{2}+\alpha_{\lambda}$, then

$$
p_{+}(\lambda, s)=\frac{\mu(\lambda)+2 s}{\mu(\lambda)+1} \text { and } p_{-}(\lambda, s)=\frac{\bar{\mu}(\lambda)+2 s}{\bar{\mu}(\lambda)+1} .
$$

Therefore, if $p_{-}(\lambda, s)<p<p_{+}(\lambda, s)$ we will be able to construct a radial supersolution for the Dirichlet problem (15) under suitable condition on $f$, just modifying the $w$ found above. Hence this bound for $p$ will be the threshold for the existence also for the Dirichlet problem.

Remark 3.1. Notice that for $s=1$,

$$
\lambda_{\alpha_{\lambda}}:=\frac{2^{2} \Gamma\left(\frac{N+2+2 \alpha_{\lambda}}{4}\right) \Gamma\left(\frac{N+2-2 \alpha_{\lambda}}{4}\right)}{\Gamma\left(\frac{N-2-2 \alpha_{\lambda}}{4}\right) \Gamma\left(\frac{N-2+2 \alpha_{\lambda}}{4}\right)}=4\left(\frac{N-2+2 \alpha_{\lambda}}{4}\right)\left(\frac{N-2-2 \alpha_{\lambda}}{4}\right)=4\left(\frac{N-2}{4}\right)^{2}-\alpha_{\lambda}^{2} .
$$

Hence $\alpha_{\lambda}= \pm \sqrt{\left(\frac{N-2}{2}\right)^{2}-\lambda}$ and $p<\frac{\frac{N+2}{2}-\alpha_{\lambda}}{\frac{N}{2}-\alpha_{\lambda}}=\frac{2+\frac{N-2}{2}-\alpha_{\lambda}}{1+\frac{N-2}{2}-\alpha_{\lambda}}=\frac{2+\alpha_{1}}{1+\alpha_{1}}=p_{+}(\lambda)$. This coincides with the nonexistence exponent defined in [5].

The first part of the main non existence result in Theorem 1.2, related to the size of the exponent of the nonlinear term is the following. 
Theorem 3.2. Assume that $s \in\left(\frac{1}{2}, 1\right)$ and $p>p_{+}(\lambda, s)$. For $\lambda>0$, problem (15) has no positive solution $u$ in the sense of Definition 2.4.

Proof. We argue by contradiction. Assume that $u$ is a positive solution to (15) in the sense of Definition 2.4 , then $u \in W_{0}^{1, p}(\Omega)$ and $\frac{u}{|x|^{2 s}} \in L^{1}(\Omega)$. By Lemma 2.9, it follows that

$$
u(x) \geqslant C|x|^{-\mu(\lambda)} \text { in } B_{r}(0) \subset \subset \Omega .
$$

The proof will be given in several steps according to the value of $p$.

First case: $p \geqslant \frac{N}{\mu(\lambda)+1}>p_{+}(\lambda, s)$.

Since $\frac{u}{|x|^{2 s}},|\nabla u|^{p} \in L^{1}\left(B_{r}(0)\right)$, then by the Poincaré-Wirtinger inequality we conclude that $u \in$ $W^{1, p}\left(L^{1}\left(B_{r}(0)\right)\right.$. Thus $u \in L^{p^{*}}\left(B_{r}(0)\right), p^{*}=\frac{N p}{N-p}$. Therefore, due to the behavior of $u$ near the origin, we conclude that $|x|^{-\mu(\lambda)} \in L^{p^{*}}\left(B_{r}(0)\right)$. Thus, $p^{*} \mu(\lambda)<N$, namely, $p<\frac{N}{\mu(\lambda)+1}$, which is a contradiction with the condition on $p$.

Second case: $2 \leqslant p<\frac{N}{\mu(\lambda)+1}$.

Since $u \in W_{0}^{1, p}(\Omega) \subset W_{0}^{1,2}(\Omega)$, then $u \in H_{0}^{s}(\Omega)$. It is well known that if $u$ is a solution in the sense of Definition 2.4, then $u$ is an entropy solution (15). Hence we can use $T_{k}(u)$, the truncation function of $u$, as a test function in (15) to conclude that

$$
\begin{aligned}
\int_{\Omega}|\nabla u|^{p} T_{k}(u) d x+\lambda \int_{\Omega} \frac{u T_{k}(u)}{|x|^{2 s}} d x & \leqslant \iint_{\mathbb{R}^{N} \times \mathbb{R}^{N}} \frac{(u(x)-u(y))\left(T_{k}(u(x))-T_{k}(u(y))\right)}{|x-y|^{N+2 s}} d y d x \\
& \leqslant\|u\|_{H_{0}^{s}(\Omega)}^{2} \leqslant C(\Omega)\|u\|_{W_{0}^{1,2}(\Omega)}^{2} .
\end{aligned}
$$

Letting $k \rightarrow \infty$ and using Fatou's lemma, we reach that

$$
\int_{\Omega} u|\nabla u|^{p} d x+\lambda \int_{\Omega} \frac{u^{2}}{|x|^{2 s}} d x \leqslant\|u\|_{H_{0}^{s}(\Omega)}^{2} \leqslant C(\Omega)\|u\|_{W_{0}^{1,2}(\Omega)}^{2} .
$$

By using the Hölder and Young inequalities we find that

$$
\int_{\Omega}|\nabla u|^{2} u d x \leqslant C_{1} \int_{\Omega}|\nabla u|^{p} u d x+C_{2} \int_{\Omega} u<\infty,
$$

and then we conclude that $u^{\frac{3}{2}} \in W_{0}^{1,2}(\Omega)$ and then $u^{\frac{3}{2}} \in H_{0}^{s}(\Omega)$. As above, using $u^{2}$ as a test function in (15) and using the fact that

$$
\left(u^{2}(x)-u^{2}(y)\right)(u(x)-u(y)) \leqslant C\left(u^{\frac{3}{2}}(x)-u^{\frac{3}{2}}(y)\right)^{2},
$$

it follows that

$$
\int_{\Omega}|\nabla u|^{p} u^{2} d x+\lambda \int_{\Omega} \frac{u^{3}}{|x|^{2 s}} d x \leqslant C|| u^{\frac{3}{2}} \|_{H_{0}^{s}(\Omega)}^{2}<\infty .
$$

Iterating the above process, it holds that

$$
\int_{\Omega}|\nabla u|^{p} u^{m} d x+\lambda \int_{\Omega} \frac{u^{m+1}}{|x|^{2 s}} d x<\infty, \text { for all } m .
$$


Choosing $(m+1) \mu(\lambda)+2 s \geqslant N$, we reach a contradiction and then the non existence result follows in this case too.

Third case: $2 s<p<2$. We follow the same idea as in the second case. Since $u \in W_{0}^{1, p}(\Omega)$, then $u \in W_{0}^{\sigma, p}(\Omega)$ for all $\sigma<1$. Setting $\sigma=\frac{2 s}{p}$, then $u \in W_{0}^{\frac{2 s}{p}, p}(\Omega)$.

We claim that if $u$ is a solution to (15), then $\int_{\Omega}|\nabla u|^{p} u^{a} d x<\infty$, for all $a>0$.

To prove the claim we begin by noticing that, since $(p-1)<1$, then for all $m>0$, we have the next algebraic inequality,

$$
(a-b)\left(a^{p m-1}-b^{p m-1}\right) \leqslant C(p, m)\left|a^{m}-b^{m}\right|^{p}, \text { for all } a, b \geqslant 0 .
$$

Thus using an approximation argument and by taking $u^{p-1}$ as a test function in (15), using the algebraic inequality (18) with $m=1$, it holds that

$$
\begin{aligned}
\int_{\Omega}|\nabla u|^{p} u^{p-1} d x+\lambda \int_{\Omega} \frac{u^{p}}{|x|^{2 s}} d x & \leqslant \iint_{D_{\Omega}} \frac{(u(x)-u(y))\left(u^{p-1}(x)-u^{p-1}(y)\right)}{|x-y|^{N+\frac{2 s}{p} p}} d x d y \\
& \leqslant C(p) \iint_{D_{\Omega}} \frac{|u(x)-u(y)|^{p}}{|x-y|^{N+\frac{2 s}{p} p} d x d y=\left.C(p)|| u\right|_{W_{0}^{\frac{2 s}{p}, p}(\Omega)} ^{p}<\infty .}
\end{aligned}
$$

Thus $\int_{\Omega}|\nabla u|^{p} u^{p-1} d x<\infty$, and then $u^{\frac{2 p-1}{p}} \in W_{0}^{1, p}(\Omega)$ and, as a consequence, $u^{\frac{2 p-1}{p}} \in W_{0}^{\frac{2 s}{p}, p}(\Omega)$.

Observe here that $\frac{2 p-1}{p}>1$. Now we set $m_{1}=\frac{2 p-1}{p}$; then choosing $u^{p m_{1}-1}$ as a test function in (15) (again using an approximation argument), it follows that

$$
\begin{aligned}
\int_{\Omega}|\nabla u|^{p} u^{p m_{1}-1} d x+\lambda \int_{\Omega} \frac{u^{p m_{1}+1}}{|x|^{2 s}} d x & \leqslant \iint_{D_{\Omega}} \frac{(u(x)-u(y))\left(u^{p m_{1}-1}(x)-u^{p m_{1}-1}(y)\right)}{|x-y|^{N+\frac{2 s}{p} p}} d x d y \\
\leqslant & C(p) \iint_{D_{\Omega}} \frac{\left|u^{m_{1}}(x)-u^{m_{1}}(y)\right|^{p}}{|x-y|^{N+\frac{2 s}{p} p}} d x d y=C(p) \| u_{\left.W_{1}^{m_{1}}\right|_{W_{(\Omega)}^{p}} ^{p}<.}^{\frac{2 s}{p}, p}<\infty .
\end{aligned}
$$

Thus $\int_{\Omega}|\nabla u|^{p} u^{p m_{1}-1} d x<\infty$ and then $u^{\frac{3 p-2}{p}} \in W_{0}^{1, p}(\Omega)$. As a consequence, $u^{\frac{3 p-2}{p}} \in W_{0}^{\frac{2 s}{p}, p}(\Omega)$. Setting $m_{j+1}=m_{j}+\left(1-\frac{1}{p}\right)$ and iterating the above process, it holds that

$$
\int_{\Omega}|\nabla u|^{p} u^{p m_{j}-1} d x<\infty
$$

for all $j$. Since $m_{j} \rightarrow \infty$ as $j \rightarrow \infty$, then we conclude that $\int_{\Omega}|\nabla u|^{p} u^{a} d x<\infty$ for all $a>0$ and the claim follows. 
Therefore, we obtain that $u^{\frac{a}{p}+1} \in W_{0}^{1, p}(\Omega)$, for all $a>0$. Thus using the local Hardy inequality in the space $W_{0}^{1, p}(\Omega)$ we reach that

$$
C(N, p) \int_{\Omega} \frac{u^{p\left(\frac{a}{p}+1\right)}}{|x|^{p}} d x \leqslant \|\left. u^{\frac{a}{p}+1}\right|_{W_{0}^{1, p}(\Omega)} ^{p}<\infty .
$$

Choosing $a \geqslant \frac{N-p(\mu(\lambda)+1)}{\mu(\lambda)}$, we reach a contradiction.

Thus the non existence result follows again in this case.

Fourth case: $p_{+}(\lambda, s)<p \leqslant 2 s$.

We deal now with the range $p_{+}(\lambda, s)<p \leqslant 2 s$, which is more involved.

Recall that $p_{+}(\lambda, s)=\frac{\mu(\lambda)+2 s}{\mu(\lambda)+1}$. Since $\lambda>0$, then $p<2 s+\mu(\lambda)$. We closely follow an argument used in [2].

Let us consider the set of functions $\mathrm{T}(\Omega)$ defined by

$$
\mathrm{T}(\Omega):=\left\{\theta \in \mathcal{C}_{0}(\Omega) \text { with } \theta \supsetneqq 0 \text { and } \operatorname{Supp} \theta \subset B_{r}(0) \subset \subset \Omega\right\} .
$$

Let $\theta \in \mathrm{T}(\Omega)$ be fixed and define $\phi_{\theta} \in H_{0}^{s}(\Omega) \cap L^{\infty}(\Omega)$, the unique solution of the problem

$$
\left\{\begin{array}{rrr}
(-\Delta)^{s} \phi_{\theta}=\theta, & \text { in } \Omega, \\
\phi_{\theta}=0, & \text { in } \mathbb{R}^{N} \backslash \Omega .
\end{array}\right.
$$

Then, according with [30], $\phi_{\theta} \simeq \delta^{s}$, where $\delta(x)$ denotes the distance to the boundary.

Using $\phi_{\theta}$ as test function in (15), it holds that

$$
\lambda \int_{\Omega} \frac{u \phi_{\theta}}{|x|^{2 s}} d x+\int_{\Omega}|\nabla u|^{p} \phi_{\theta} d x<\int_{\Omega} u(-\Delta)^{s} \phi_{\theta} d x=\int_{\Omega} u \theta d x .
$$

Now, consider $\psi_{\theta} \in W_{l o c}^{1, p}(\Omega)$ to be the unique solution to the problem

$$
\left\{\begin{aligned}
-\operatorname{div}\left(\phi_{\theta}^{a}\left|\nabla \psi_{\theta}\right|^{p-2} \nabla \psi_{\theta}\right)=\theta, & \text { in } \Omega, \\
\psi_{\theta}=0, & \text { on } \partial \Omega,
\end{aligned}\right.
$$

where $a<\frac{p-1}{s}$. Existence of $\psi_{\theta}$ will be proved in Lemma 3.3 below.

Going back to (21) and using Young's inequality, we have that

$$
\begin{aligned}
\lambda \int_{\Omega} \frac{u \phi_{\theta}}{|x|^{2 s}} d x+\int_{\Omega}|\nabla u|^{p} \phi_{\theta} d x & \leqslant \int_{\Omega} u \theta d x=\int_{\Omega} u\left(-\operatorname{div}\left(\phi_{\theta}^{a}\left|\nabla \psi_{\theta}\right|^{p-2} \nabla \psi_{\theta}\right)\right) d x \\
& \leqslant \int_{\Omega} \phi_{\theta}^{a}|\nabla u|\left|\nabla \psi_{\theta}\right|^{p-1} d x \\
& \leqslant \frac{1}{2} \int_{\Omega} \phi_{\theta}|\nabla u|^{p} d x+C_{2} \int_{\Omega} \phi_{\theta}^{(a-1) p^{\prime}+1}\left|\nabla \psi_{\theta}\right|^{p} d x .
\end{aligned}
$$

Thus, we get

$$
\lambda \int_{\Omega} \frac{u \phi_{\theta}}{|x|^{2 s}} d x \leqslant C_{2} \int_{\Omega} \phi_{\theta}^{(a-1) p^{\prime}+1}\left|\nabla \psi_{\theta}\right|^{p} d x
$$

with $C_{2}$ depending only on $p$. Due to the behavior of $u$ near the origin, we get

$$
\lambda \int_{B_{r}(0)} \frac{\phi_{\theta}}{|x|^{2 s+\mu(\lambda)}} d x \leqslant C_{2} \int_{\Omega} \phi_{\theta}^{(a-1) p^{\prime}+1}\left|\nabla \psi_{\theta}\right|^{p} d x .
$$


Setting

$$
\mathrm{Q}(\theta):=\frac{\int_{\Omega} \phi_{\theta}^{(a-1) p^{\prime}+1}\left|\nabla \psi_{\theta}\right|^{p}}{\int_{B_{r}(0)} \frac{\phi_{\theta}}{|x|^{2 s+\mu(\lambda)}} d x}
$$

then $\mathrm{Q}(\theta)=\mathrm{Q}(\mu \theta)$ for all $\mu>0$. Thus

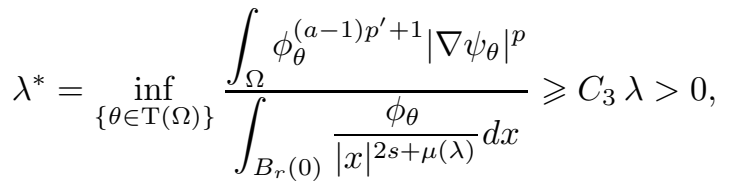

where $C_{3}$ depends only on $p$. Notice that, going back to inequality (23) and since $\lambda>0$ is fixed, then if $\int_{\Omega} \phi_{\theta}^{(a-1) p^{\prime}+1}\left|\nabla \psi_{\theta}\right|^{p}<\infty$, it holds that $\int_{B_{r}(0)} \frac{\phi_{\theta}}{|x|^{2 s+\mu(\lambda)}} d x<\infty$ which will be the key in order to get the desired contradiction.

Notice that, using a suitable approximation and density argument, inequality (23) holds for all $\theta \in L^{1}(\Omega)$ with $\operatorname{Supp}(\theta) \subset \subset \Omega$ if, in addition, we can show that $\int_{\Omega} \phi_{\theta}^{(a-1) p^{\prime}+1}\left|\nabla \psi_{\theta}\right|^{p} d x<\infty$. This will be the main idea in order to get the desired results.

Without loss of generality we can assume that $B_{1}(0) \subset \subset \Omega$. Consider

$$
\theta(x)=\left(\frac{1}{|x|^{m}}-1\right)+\text { with } \max \{2 s, N-\mu(\lambda)\}<m<N
$$

and define $\phi_{\theta}$, the unique solution to problem (20) (that can be considered in a very weak sense or entropy sense). Then

$$
\phi_{\theta} \simeq \frac{\delta^{s}(x)}{|x|^{m-2 s}}
$$

Since $m-2 s<N-p$ and $a<\frac{p-1}{s}<1$, then the weight $\frac{1}{|x|^{a(m-2 s)}}$ is admissible in the sense of Caffarelli-Kohn-Nirenberg inequalities.

We claim that the auxiliary problem $(22)$ has a solution $\psi_{\theta}$ such that, under the above condition on $m$ and $p$, we have $\int_{\Omega} \phi_{\theta}^{(a-1) p^{\prime}+1}\left|\nabla \psi_{\theta}\right|^{p}<\infty$. Since Supp $\theta \subset \subset \Omega$ and $\theta, \phi_{\theta}$ are only singular at the origin, then we have to show that $\int_{B_{r}(0)} \phi_{\theta}^{(a-1) p^{\prime}+1}\left|\nabla \psi_{\theta}\right|^{p}<\infty$.

Define $\widetilde{\psi_{\theta}}$ to be the unique solution to the problem

$$
\left\{\begin{aligned}
-\operatorname{div}\left(\frac{1}{|x|^{a(m-2 s)}}\left|\nabla \widetilde{\psi_{\theta}}\right|^{p-2} \nabla \widetilde{\psi_{\theta}}\right) & =\frac{1}{|x|^{m}}, & & \text { in } \Omega, \\
\widetilde{\psi_{\theta}} & =0, & & \text { on } \partial \Omega .
\end{aligned}\right.
$$

By a direct computation we can show that, as $x \rightarrow 0, \widetilde{\psi_{\theta}} \simeq \frac{C(\Omega)}{|x| \frac{(1-a)(m-2 s)}{p-1}+\frac{2 s-p}{p-1}}$ and

$$
\left|\nabla \widetilde{\psi_{\theta}}\right| \simeq \frac{C(\Omega)}{|x|^{\frac{(1-a)(m-2 s)}{p-1}+\frac{2 s-1}{p-1}}} .
$$


Then

$$
\left(\frac{1}{|x|^{m-2 s}}\right)^{(a-1) p^{\prime}+1}\left|\nabla \widetilde{\psi_{\theta}}\right|^{p} \approx \frac{C(\Omega)}{|x|^{(m-2 s)+p^{\prime}(2 s-1)}},
$$

in a neighborhood of the origin. Since $m>N-\mu(\lambda)$, then $(m-2 s)+p^{\prime}(2 s-1)<N$, if and only if $p>p_{+}(\lambda, s)$.

Thus

$$
\int_{\Omega}\left(\frac{1}{|x|^{m-2 s}}\right)^{(a-1) p^{\prime}+1}\left|\nabla \widetilde{\psi_{\theta}}\right|^{p}<\infty \text { and then } \int_{B_{r}(0)} \phi_{\theta}^{(a-1) p^{\prime}+1}\left|\nabla \psi_{\theta}\right|^{p}<\infty .
$$

However, notice that, by a direct computation,

$$
\int_{B_{r}(0)} \frac{\phi_{\theta}}{|x|^{2 s+\mu(\lambda)}} d x=\infty
$$

which is a contradiction.

To finish with the proof of Theorem 3.2 we need to show the existence of a solution for the $p$ Laplacian weighted problem (22), as was stated in the fourth case considered above. This is the content of the following lemma.

Lemma 3.3. Let $\Omega \subset \mathbb{R}^{N}, N \geqslant 2$, be a bounded domain with boundary $\partial \Omega$ of class $\mathcal{C}^{2}$. Assume that $\theta \in \mathrm{T}(\Omega)$ defined in (19) and let $\phi_{\theta} \in H_{0}^{s}(\Omega) \cap L^{\infty}(\Omega)$ be the solution of

$$
\left\{\begin{array}{rrr}
(-\Delta)^{s} \phi_{\theta}=\theta, & \text { in } \Omega, \\
\phi_{\theta}=0, & \text { in } \mathbb{R}^{N} \backslash \Omega .
\end{array}\right.
$$

Suppose that $1<p \leqslant 2 s$ and $a<\frac{p-1}{s}$. Then there exists $\psi_{\theta} \in W_{l o c}^{1, p}(\Omega)$ distributional solution of

$$
\left\{\begin{aligned}
-\operatorname{div}\left(\phi_{\theta}^{a}\left|\nabla \psi_{\theta}\right|^{p-2} \nabla \psi_{\theta}\right)=\theta, & \text { in } \Omega, \\
\psi_{\theta}=0, & \text { on } \partial \Omega .
\end{aligned}\right.
$$

Moreover $\phi_{\theta} \in W_{0}^{1, p}\left(\delta^{a s}(x) d x, \Omega\right)$ where $W_{0}^{1, p}\left(\delta^{a s}(x) d x, \Omega\right)$ is the completion of $\mathcal{C}_{0}^{\infty}(\Omega)$ with respect to the norm

$$
\|\phi\|_{1}^{p}:=\int_{\Omega} \delta^{a s}(x)|\nabla v|^{p} d x \text { where } \delta(x):=\operatorname{dist}(x, \partial \Omega) .
$$

Before proving Lemma 3.3, let us recall the next weighted Hardy inequality proved in [27], Theorem 1.6 .

Theorem 3.4. Assume that $\Omega \subset \mathbb{R}^{N}, N \geqslant 2$, is a bounded regular domain and let $0<\sigma<p-1$. Then, there exists a positive constant $C=C(\Omega, p, \sigma)$ such that for all $v \in \mathcal{C}_{0}^{\infty}(\Omega)$, we have

$$
\int_{\Omega} \delta^{\sigma-p}(x)|v|^{p} d x \leqslant C \int_{\Omega} \delta^{\sigma}(x)|\nabla v|^{p} d x .
$$

Proof of Lemma 3.3. By the results of [30], we know that

$$
C_{1} \delta^{s}(x) \leqslant \phi_{\theta}(x) \leqslant C_{2} \delta^{s}(x), \quad \forall x \in \Omega,
$$

with $C_{1}, C_{2}>0$. For $n \in \mathbb{N}$, we consider the approximate problems

$$
\left\{\begin{aligned}
-\operatorname{div}\left(\left(\phi_{\theta}+\frac{1}{n}\right)^{a}\left|\nabla \psi_{n}\right|^{p-2} \psi_{n}\right) & =\theta, \quad \text { in } \Omega, \\
\psi_{n} & =0, \quad \text { on } \partial \Omega .
\end{aligned}\right.
$$


It is clear that the existence of $\psi_{n}$ follows using classical variational argument where we obtain also that $\psi_{n} \in W_{0}^{1, p}(\Omega) \cap L^{\infty}(\Omega)$. Using $\psi_{n}$ as test function in (27), we get

$$
C_{1} \int_{\Omega} \delta^{a s}(x)\left|\nabla \psi_{n}\right|^{p} d x \leqslant \int_{\Omega} \theta \psi_{n}(x) d x .
$$

Hence by Theorem 3.4 and choosing $\sigma=a s \in(p-1, p)$, it holds that

$$
C_{1} \int_{\Omega} \delta^{a s}(x)\left|\nabla \psi_{n}\right|^{p} d x \leqslant\|\theta\|_{L^{p^{\prime}}(\Omega)}\|w\|_{L^{p}(\Omega)} \leqslant C\|\theta\|_{L^{p^{\prime}}(\Omega)}\left(\int_{\Omega} \delta^{a s}(x)\left|\nabla \psi_{n}\right|^{p} d x\right)^{\frac{1}{p}} .
$$

Thus $\int_{\Omega} \delta^{a s}(x)\left|\nabla \psi_{n}\right|^{p} d x \leqslant C$ for all $n$. Hence $\left\{\psi_{n}\right\}_{n}$ is bounded in the space $W_{0}^{1, p}\left(\delta^{a s}(x) d x, \Omega\right)$. Therefore, using again Theorem 3.4, it holds that $\int_{\Omega} \frac{\left|\psi_{n}\right|^{p}}{\delta^{p-a s}} d x \leqslant C$, for all $n$.

Then, up to a subsequence, we get the existence of $\psi \in W_{0}^{1, p}\left(\delta^{a s}(x) d x, \Omega\right)$ such that $\psi_{n} \rightarrow \psi$ weakly in $W_{0}^{1, p}\left(\delta^{a s}(x) d x, \Omega\right)$, and then $\psi_{n} \rightarrow \psi$ in $L_{l o c}^{\sigma}(\Omega)$ for all $1 \leqslant \sigma<p^{*}$ and $\psi_{n} \rightarrow \psi$ a.e. in $\Omega$.

Using Vitali's lemma we can prove that $\psi_{n} \rightarrow \psi$ strongly in $L^{p}(\Omega)$.

It is not difficult to show that $\psi$ is a distributional solution to problem (26).

Let show that $\psi_{n} \rightarrow \psi$ strongly in $W_{0}^{1, p}\left(\Omega, \delta^{a s}(x) d x\right)$.

Using $\left(\psi_{n}-\psi\right)$ as test function in (27) and having into account that

$$
\int_{\Omega} \theta\left(\psi_{n}-\psi\right) d x \rightarrow 0 \text { as } n \rightarrow \infty,
$$

it follows that

$$
C_{1} \int_{\Omega} \delta^{a s}(x)\left|\nabla \psi_{n}\right|^{p-2} \nabla \psi_{n} \nabla\left(\psi_{n}-\psi\right) d x \leqslant o(1)
$$

Since

$$
\int_{\Omega} \delta^{a s}(x)\left|\nabla \psi_{n}\right|^{p-2} \nabla \psi_{n} \nabla\left(\psi_{n}-\psi\right) d x=\int_{\Omega} \delta^{a s}(x)\left(\left|\nabla \psi_{n}\right|^{p-2} \nabla \psi_{n}-|\nabla \psi|^{p-2} \nabla \psi\right) \nabla\left(\psi_{n}-\psi\right) d x+o(1),
$$

then the result follows. In a similar way one can show the uniqueness of the solution in the space $W_{0}^{1, p}\left(\delta^{a s}(x) d x, \Omega\right)$.

To finish this section we prove the next non existence result for $\mu$ large.

Theorem 3.5. Assume that $s \in\left(\frac{1}{2}, 1\right)$, then there exists $\mu^{*}>0$ such that if $\mu>\mu^{*}$, the problem (15) has no positive solution $u$ in the sense of Definition 2.4.

Proof. We follow closely the proof of the fourth case in Theorem 3.2, see also [2]. Assume that $u$ is a positive solution to problem (15) in the sense of Definition 2.4. Let $\theta \in \mathcal{C}_{0}^{\infty}(\Omega)$ be a nonnegative fixed function and define $\phi_{\theta} \in H_{0}^{s}(\Omega) \cap L^{\infty}(\Omega)$, the unique solution of the problem

$$
\left\{\begin{array}{rr}
(-\Delta)^{s} \phi_{\theta}=\theta, \quad \text { in } \Omega, \\
\phi_{\theta}=0, \quad \text { in } \mathbb{R}^{N} \backslash \Omega .
\end{array}\right.
$$

Since $\theta$ is bounded, according with [30], then $\phi_{\theta} \simeq \delta^{s}$.

Using $\phi_{\theta}$ as test function in (15), it holds that

$$
\mu \int_{\Omega} f(x) \phi_{\theta}(x) d x+\int_{\Omega}|\nabla u|^{p} \phi_{\theta} d x \leqslant \int_{\Omega} u(-\Delta)^{s} \phi_{\theta} d x=\int_{\Omega} u \theta d x .
$$


Now, define $\psi_{\theta} \in W_{l o c}^{1, p}(\Omega)$ as the unique solution to the problem

$$
\left\{\begin{aligned}
-\operatorname{div}\left(\phi_{\theta}\left|\nabla \psi_{\theta}\right|^{p-2} \nabla \psi_{\theta}\right) & =\theta, \quad \text { in } \Omega, \\
\psi_{\theta} & =0, \quad \text { on } \partial \Omega .
\end{aligned}\right.
$$

Notice that the existence of $\psi_{\theta}$ follows using the same kind of estimates as in the proof of Lemma 3.3. Hence

$$
\mu \int_{\Omega} f(x) \phi_{\theta}(x) d x+\int_{\Omega}|\nabla u|^{p} \phi_{\theta} d x \leqslant \int_{\Omega} u \theta d x=-\operatorname{div}\left(\phi_{\theta}\left|\nabla \psi_{\theta}\right|^{p-2} \nabla \psi_{\theta}\right) .
$$

Thus

$$
\begin{aligned}
\mu \int_{\Omega} f(x) \phi_{\theta}(x) d x+\int_{\Omega}|\nabla u|^{p} \phi_{\theta} d x & \leqslant \int_{\Omega} \phi_{\theta}\left|\nabla \psi_{\theta}\right|^{p-1}|\nabla u| d x \phi_{\theta} d x \\
& \leqslant \varepsilon \int_{\Omega}|\nabla u|^{p} \phi_{\theta} d x+C(\varepsilon) \int_{\Omega} \phi_{\theta}\left|\nabla \psi_{\theta}\right|^{p} d x .
\end{aligned}
$$

Choosing $\varepsilon$ small it holds that

$$
\mu \int_{\Omega} f(x) \phi_{\theta}(x) d x \leqslant C(\varepsilon) \int_{\Omega} \phi_{\theta}\left|\nabla \psi_{\theta}\right|^{p} d x
$$

Thus

$$
\mu \leqslant \inf _{\left\{\theta \in \mathcal{C}_{0}^{\infty}(\Omega), \theta \geqslant 0\right\}} \frac{\int_{\Omega} \phi_{\theta}\left|\nabla \psi_{\theta}\right|^{p}}{\int_{\Omega} f(x) \phi_{\theta} d x}:=\mu^{*}
$$

and the result follows.

\section{EXISTENCE RESUlT}

In this section we consider the problem

$$
\left\{\begin{aligned}
(-\Delta)^{s} u & =\lambda \frac{u}{|x|^{2 s}}+|\nabla u|^{p}+\mu f & & \text { in } \Omega, \\
u & =0 & & \text { in } \mathbb{R}^{N} \backslash \Omega, \\
u & >0 & & \text { in } \Omega,
\end{aligned}\right.
$$

where $0<\lambda<\Lambda_{N, s}, \mu>0, s \in\left(\frac{1}{2}, 1\right), p<p_{+}(\lambda, s)<2 s$ and $f \in L^{\sigma}(\Omega)$ for some convenient $\sigma>1$. The main goal of this section is to show that, under additional hypotheses on $f$, we are able to build a suitable supersolution and then by a monotonicity argument, to prove the existence of a minimal positive solution.

Before the statement of the existence result of this section, let us recall a compactness result obtained in [17] and the comparison result that will be used in this section.

Theorem 4.1. Suppose that $s \in\left(\frac{1}{2}, 1\right)$ and let $f \in \mathfrak{M}(\Omega)$, a Radon measure. Then the problem

$$
\left\{\begin{array}{rll}
(-\Delta)^{s} v= & \text { in } \Omega \\
u=0 & \text { in } \mathbb{R}^{N} \backslash \Omega,
\end{array}\right.
$$

has a unique weak solution in the sense of Definition 2.2 such that, 
(1) $|\nabla v| \in M^{p_{*}, \infty}(\Omega)$, the Marcinkiewicz space, with

$$
p_{*}=\frac{N}{N-2 s+1}
$$

and as a consequence $v \in W_{0}^{1, q}(\Omega)$ for all $q<p_{*}$. Moreover

$$
\|v\|_{W_{0}^{1, q}(\Omega)} \leqslant C(N, q, \Omega)\|f\|_{\mathfrak{M}(\Omega)} .
$$

(2) For $f \in L^{1}(\Omega)$, setting $T: L^{1}(\Omega) \rightarrow W_{0}^{1, q}(\Omega)$, with $T(f)=v$, then $T$ is a compact operator.

The next comparison principle is proved in [6], and extends the one proved in [8] in the local case.

Theorem 4.2. (Comparison Principle). Let $g \in L^{1}(\Omega)$ be a nonnegative function and consider $p_{*}$ defined in (36). Assume that for all $\xi_{1}, \xi_{2} \in \mathbb{R}^{N}$,

$$
H: \Omega \times \mathbb{R}^{N} \rightarrow \mathbb{R}^{+} \text {satisfies }\left|H\left(x, \xi_{1}\right)-H\left(x, \xi_{2}\right)\right| \leqslant C b(x)\left|\xi_{1}-\xi_{2}\right|
$$

where $b \in L^{\sigma}(\Omega)$ for some $\sigma>\frac{N}{2 s-1}$. Consider $w_{1}, w_{2}$ two positive functions such that $w_{1}, w_{2} \in$ $W^{1, p}(\Omega)$ for all $p<p_{*},(-\Delta)^{s} w_{1},(-\Delta)^{s} w_{2} \in L^{1}(\Omega), w_{1} \leqslant w_{2}$ in $\mathbb{R}^{N} \backslash \Omega$ and

$$
\begin{cases}(-\Delta)^{s} w_{1} \leqslant H\left(x, \nabla w_{1}\right)+g & \text { in } \Omega, \\ (-\Delta)^{s} w_{2} \geqslant H\left(x, \nabla w_{2}\right)+g & \text { in } \Omega .\end{cases}
$$

Then, $w_{2} \geqslant w_{1}$ in $\Omega$.

As a consequence, we have the following

Theorem 4.3. Assume that $g \in L^{1}(\Omega)$ is a nonnegative function. Let $w_{1}, w_{2}$ be two nonnegative functions such that $w_{1}, w_{2} \in W^{1, p}(\Omega)$ for some $1 \leqslant q<p_{*},(-\Delta)^{s} w_{1},(-\Delta)^{s} w_{2} \in L^{1}(\Omega), w_{1} \leqslant w_{2}$ in $\mathbb{R}^{N} \backslash \Omega$ and

$$
\begin{cases}(-\Delta)^{s} w_{1} \leqslant\left|\nabla w_{1}\right|^{q}+g & \text { in } \Omega \\ (-\Delta)^{s} w_{2} \geqslant\left|\nabla w_{2}\right|^{q}+g & \text { in } \Omega .\end{cases}
$$

Then, $w_{2} \geqslant w_{1}$ in $\mathbb{R}^{N}$.

Since we will use the representation formula for the solution of problem (35), then we recall the main properties of the Green function associated to the fractional laplacian. The proof can be found in [11], [12] [13], using a probabilistic approach.

Lemma 4.4. Let $\mathcal{G}_{s}$ be the Green kernel of $(-\Delta)^{s}$ and suppose that $s \in\left(\frac{1}{2}, 1\right)$, then

$$
\mathcal{G}_{s}(x, y) \leqslant C_{1} \min \left\{\frac{1}{|x-y|^{N-2 s}}, \frac{\delta^{s}(x)}{|x-y|^{N-s}}, \frac{\delta^{s}(y)}{|x-y|^{N-s}}\right\},
$$

and

$$
\left|\nabla_{x} \mathcal{G}_{s}(x, y)\right| \leqslant C_{2} \mathcal{G}_{s}(x, y) \max \left\{\frac{1}{|x-y|}, \frac{1}{\delta(x)}\right\} .
$$

4.1. A radial supersolution. We will start by building a radial supersolution with an appropriate regularity.

We begin with the case $p_{-}(\lambda, s)<q<p_{+}(\lambda, s)<2 s$. Let $w_{1}$ be the solution to the equation (16) obtained in the first section. Recall that $w_{1}(x)=\frac{A}{|x|^{\theta_{0}}}$ with $\theta_{0}=\frac{N-2 s}{2}-\beta$ and

$$
(-\Delta)^{s} w_{1}(x)-\lambda \frac{w_{1}}{|x|^{2 s}}=\frac{A\left(\gamma_{\beta}-\lambda\right)}{|x|^{\theta_{0}+2 s}}=\frac{A^{p-1}\left|\theta_{0}\right|^{p}}{|x|^{\theta_{0}+2 s}} \text {. }
$$


By the definition of $\gamma_{\beta}$ given in (17), it holds that $\left(\gamma_{\beta}-\lambda\right)>0$ if and only if $\theta_{0} \in(\mu(\lambda), \bar{\mu}(\lambda))$. It is clear that, in order to get

$$
\frac{A\left(\gamma_{\beta}-\lambda\right)}{|x|^{\theta_{0}+2 s}} \geqslant\left|\nabla w_{1}\right|^{p} \text { in } \Omega
$$

we need that $\theta_{0}<p_{+}(\lambda, s)$.

Now, fix $\theta \in(\mu(\lambda), \bar{\mu}(\lambda))$ close to $\mu(\lambda)$ such that if we set $w(x)=A|x|^{-\theta}, \quad A>0$, then

$$
(-\Delta)^{s} w(x)=\lambda \frac{w}{|x|^{2 s}}+\frac{C(A, \lambda)}{|x|^{2 s+\theta}} .
$$

It is clear that $|\nabla w(x)|=\frac{A \theta}{|x|^{\theta+1}}$, hence $\frac{C(A, \lambda)}{|x|^{2 s+\theta}} \geqslant|\nabla w(x)|^{p}$ in a neighborhood of the origin if $\theta+2 s>q(\theta+1)$. Thus $\theta<\frac{2 s-p}{p-1}$.

Hence we can fix $\alpha>0$ such that $\theta<\frac{2 s-\alpha}{\alpha-1}$ and $p_{-}(\lambda, s)<p<\alpha<p_{+}(\lambda, s)$. From now on, we fix $\alpha$ such that the above construction holds.

Notice that, since $p<p_{+}(\lambda, s)$, then $\frac{2 s-p}{p-1}>\mu(\lambda)$. Also, since $p_{-}(\lambda, s)<p$, then $\frac{2 s-p}{p-1}<\bar{\mu}(\lambda)$.

Clearly, if $f \leqslant \frac{1}{|x|^{2 s+\theta}}$, then $w_{1}$ is a supersolution to problem (34) for $\mu<\mu^{*}$.

We analyze now some properties of this supersolution.

Recall that $\mathcal{G}_{s}$ is the Green kernel of $(-\Delta)^{s}$ and define

$$
K(y)=\int_{\Omega} \frac{w^{\alpha-1}(x) \mathcal{G}_{s}(x, y)}{|x-y|^{\alpha}} d x,
$$

where $\alpha<2 s$ to be chosen later. We claim that $K \in L^{\infty}(\Omega)$. To show the claim, we observe that

$$
K(y) \leqslant C \int_{\Omega} \frac{1}{|x|^{\theta(\alpha-1)}|x-y|^{N-2 s+\alpha}} d x .
$$

Since $\theta<\frac{2 s-\alpha}{\alpha-1}$ and $\alpha<2 s$, it holds that $\frac{N}{2 s-\alpha}<\frac{N}{\theta(\alpha-1)}$. Hence we get the existence of $\frac{N}{2 s-\alpha}<\sigma<$ $\frac{N}{\theta(\alpha-1)}$ such that $\frac{1}{|x|^{\theta(\alpha-1)}} \in L^{\sigma}(\Omega)$.

Using Hölder inequality, we have that

$$
\left.K(y) \leqslant C\left(\int_{\Omega} \frac{1}{|x|^{\theta(\alpha-1)}}\right)^{\sigma} d x\right)^{\frac{1}{\sigma}}\left(\int_{\Omega} \frac{1}{|x-y|^{\sigma^{\prime}(N-2 s+\alpha)}} d x\right)^{\frac{1}{\sigma^{\prime}}} .
$$

Since $\sigma^{\prime}(N-2 s+\alpha)<N$, then $K(y) \leqslant C$ for all $y \in \Omega$ and the claim follows.

Consider now $\psi$ to be the unique solution to the problem

$$
\left\{\begin{array}{rlrl}
(-\Delta)^{s} \psi & =\frac{w^{\alpha-1}(x)}{\delta^{\alpha}(x)}=\frac{A^{\alpha-1}}{|x|^{\theta(\alpha-1)} \delta^{\alpha}(x)} & & \text { in } \Omega, \\
\psi & & \text { in } \mathbb{R}^{N} \backslash \Omega .
\end{array}\right.
$$

Since $\theta(\alpha-1)<2 s$, as in [6], we can prove that $\psi \in L^{\infty}(\Omega)$.

We are now in position to state the main existence result.

Theorem 4.5. Assume $f \in L^{\infty}(\Omega)$ and suppose that $1<p<p_{+}(\lambda, s)<2 s$. Then problem (34) has a solution $u$ such that $u \in W_{0}^{1, p}(\Omega)$. 
Proof. We divide the proof into two parts according to the value of $p$.

First case: $p_{-}(\lambda, s)<p<p_{+}(\lambda, s)$.

Let $w$ be the supersolution obtained in the previous computation, then $w \in W^{1, \alpha}(\Omega)$. Consider $u_{n}$ to be the unique solution to the approximating problem

$$
\left\{\begin{aligned}
(-\Delta)^{s} u_{n} & =\frac{\left|\nabla u_{n}\right|^{p}}{1+\frac{1}{n}\left|\nabla u_{n}\right|^{p}}+\lambda \frac{u_{n}}{|x|^{2 s}}+\mu f & & \text { in } \Omega, \\
u_{n} & =0 & & \text { in } \mathbb{R}^{N} \backslash \Omega .
\end{aligned}\right.
$$

By the comparison principle in Theorem 4.2, it follows that $u_{n} \leqslant u_{n+1} \leqslant w$ for all $n$. Since $w \in L^{p^{*}}(\Omega)$, then there exists $u$ such that $u_{n} \uparrow u$ strongly in $L^{p^{*}}(\Omega)$. Define

$$
g_{n}(x)=\frac{\left|\nabla u_{n}\right|^{p}}{1+\frac{1}{n}\left|\nabla u_{n}\right|^{p}}+\lambda \frac{u_{n}}{|x|^{2 s}}+\mu f,
$$

since $u_{n} \leqslant w$, using the positive first eigenfunction of the fractional laplacian $\varphi_{1}$ as test function in (43) and using the fact that $\varphi_{1} \simeq \delta^{s}$, it holds that

$$
\int_{\Omega} g_{n}(x) \delta^{s}(x) d x \leqslant \lambda_{1} \int_{\Omega} u_{n} \varphi_{1} \leqslant C(\Omega) \int_{\Omega} w \delta^{s}(x) d x \leqslant C \text { for all } n .
$$

We claim that the sequence $\left\{u_{n}\right\}_{n}$ is bounded in $W_{0}^{1, \alpha}(\Omega)$ where $\alpha<2 s$ is chosen as in the definition of the supersolution.

We follow the same ideas as in [6]. We have that

$$
u_{n}(x)=\int_{\Omega} \mathcal{G}_{s}(x, y) g_{n}(y) d y .
$$

Hence

$$
\left|\nabla u_{n}(x)\right| \leqslant \int_{\Omega}\left|\nabla_{x} \mathcal{G}_{s}(x, y)\right| g_{n}(y) d y .
$$

Fix $1<\alpha<2 s$ and define $h(x, y)=\max \left\{\frac{1}{|x-y|}, \frac{1}{\delta(x)}\right\}$. Then,

$$
\begin{aligned}
\left|\nabla u_{n}(x)\right|^{\alpha} & \leqslant\left(\int_{\Omega}\left|\nabla_{x} \mathcal{G}_{s}(x, y)\right| g_{n}(y) d y\right)^{\alpha} \leqslant\left(\int_{\Omega} h(x, y) \mathcal{G}_{s}(x, y) g_{n}(y) d y\right)^{\alpha} \\
& \leqslant\left(\int_{\Omega}(h(x, y))^{\alpha} \mathcal{G}_{s}(x, y) g_{n}(y) d y\right)\left(\int_{\Omega} \mathcal{G}_{s}(x, y) g_{n}(y) d y\right)^{\alpha-1} \\
& \leqslant\left(\int_{\Omega}\left(h^{\alpha}(x, y) \mathcal{G}_{s}(x, y) g_{n}(y) d y\right) u_{n}^{\alpha-1}(x)\right. \\
& \leqslant \int_{\Omega}\left(h^{\alpha}(x, y) \mathcal{G}_{s}(x, y) g_{n}(y) d y\right) w^{\alpha-1}(x) \\
& \leqslant \int_{\Omega}\left(h^{\alpha}(x, y) \mathcal{G}_{s}(x, y)\left|\nabla u_{n}(y)\right|^{p} d y\right) w^{\alpha-1}(x)+\lambda \int_{\Omega}\left(h^{\alpha}(x, y) \mathcal{G}_{s}(x, y) \frac{u_{n}(y)}{|y|^{2 s}} d y\right) w^{\alpha-1}(x) \\
& +\mu \int_{\Omega}\left(h^{\alpha}(x, y) \mathcal{G}_{s}(x, y) f(y) d y\right) w^{\alpha-1}(x) .
\end{aligned}
$$


Thus

$$
\begin{aligned}
\int_{\Omega}\left|\nabla u_{n}\right|^{\alpha} d x & \leqslant \int_{\Omega}\left|\nabla u_{n}(y)\right|^{p}\left(\int_{\Omega} h^{\alpha}(x, y) \mathcal{G}_{s}(x, y) w^{\alpha-1}(x)\right) d y \\
& +\lambda \int_{\Omega} \frac{w(y)}{|y|^{2 s}}\left(\int_{\Omega} h^{\alpha}(x, y) \mathcal{G}_{s}(x, y) w^{\alpha-1}(x) d x\right) d y \\
& +\mu \int_{\Omega} f(y)\left(\int_{\Omega} h^{\alpha}(x, y) \mathcal{G}_{s}(x, y) w^{\alpha-1}(x) d x\right) d y \\
& \equiv J_{1}+J_{2}+J_{3} .
\end{aligned}
$$

Observing that $h^{\alpha}(x, y) \leqslant \frac{1}{|x-y|^{\alpha}}+\frac{1}{\delta(x)^{\alpha}}$, then

$$
\begin{aligned}
J_{1} & \leqslant \int_{\Omega}\left|\nabla u_{n}(y)\right|^{p}\left(\int_{\Omega} \frac{w^{\alpha-1}(x) \mathcal{G}_{s}(x, y)}{|x-y|^{\alpha}} d x\right) d y+\int_{\Omega}\left|\nabla u_{n}(y)\right|^{p}\left(\int_{\Omega} \frac{w^{\alpha-1}(x) \mathcal{G}_{s}(x, y)}{\delta^{\alpha}(x)} d x\right) d y \\
& \leqslant \int_{\Omega}\left|\nabla u_{n}(y)\right|^{p}\left(\int_{\Omega} \frac{w^{\alpha-1}(x) \mathcal{G}_{s}(x, y)}{|x-y|^{\alpha}} d x\right) d y+\int_{\Omega}\left|\nabla u_{n}(y)\right|^{p} \psi(y) d y .
\end{aligned}
$$

By using the hypothesis on $w$, we reach that

$$
\begin{aligned}
J_{1} & \leqslant C \int_{\Omega}\left|\nabla u_{n}(y)\right|^{p} d y+\left(\int_{\Omega}\left|\nabla u_{n}(y)\right|^{\alpha} d y\right)^{\frac{p}{\alpha}}\left(\int_{\Omega} \psi \psi^{\frac{\alpha}{p-\alpha}} d y\right)^{\frac{\alpha-p}{\alpha}} \\
& \leqslant C_{1} \int_{\Omega}\left|\nabla u_{n}(y)\right|^{p} d y+C_{2}\left(\int_{\Omega}\left|\nabla u_{n}(y)\right|^{\alpha} d y\right)^{\frac{p}{\alpha}} .
\end{aligned}
$$

We deal now with $J_{2}$.

$$
\begin{aligned}
J_{2} & \leqslant \int_{\Omega} \frac{w(y)}{|y|^{2 s}}\left(\int_{\Omega} \frac{w^{\alpha-1}(x) \mathcal{G}_{s}(x, y)}{|x-y|^{\alpha}} d x\right) d y+\int_{\Omega} \frac{w(y)}{|y|^{2 s}}\left(\int_{\Omega} \frac{w^{\alpha-1}(x) \mathcal{G}_{s}(x, y)}{\delta^{\alpha}(x)} d x\right) d y \\
& \leqslant \int_{\Omega} \frac{w(y)}{|y|^{2 s}}\left(\int_{\Omega} \frac{w^{\alpha-1}(x) \mathcal{G}_{s}(x, y)}{|x-y|^{\alpha}} d x\right) d y+\int_{\Omega} \frac{w(y)}{|y|^{2 s}} \psi(y) d y \leqslant C \int_{\Omega} \frac{w(y)}{|y|^{2 s}} d y \leqslant C .
\end{aligned}
$$

For $J_{3}$, we have

$$
J_{3} \leqslant \int_{\Omega} f(y)\left(\int_{\Omega} \frac{w^{\alpha-1}(x) \mathcal{G}_{s}(x, y)}{|x-y|^{\alpha}} d x\right) d y+\int_{\Omega} f(y) \psi(y) d y .
$$

Hence,

$$
\begin{aligned}
J_{3} & \leqslant C \int_{\Omega} f(y) d y+\left(\int_{\Omega} f^{\frac{N}{p^{\prime}(2 s-1)}} d y\right)^{\frac{p^{\prime}(2 s-1)}{N}}\left(\int_{\Omega} \psi^{\frac{N}{N-p^{\prime}(2 s-1)}} d y\right)^{\frac{N-p^{\prime}(2 s-1)}{N}} \\
& \leqslant C .
\end{aligned}
$$

Therefore we conclude that

$$
\int_{\Omega}\left|\nabla u_{n}(x)\right|^{\alpha} d x \leqslant C_{1} \int_{\Omega}\left|\nabla u_{n}(x)\right|^{p} d x+C_{2} .
$$


Choosing $\alpha>q$ and by Hölder inequality, we obtain that

$$
\int_{\Omega}\left|\nabla u_{n}(x)\right|^{\alpha} d x \leqslant C \text { for all } n .
$$

As a consequence we get that the sequence $\left\{g_{n}\right\}_{n}$ is bounded in $L^{1+\varepsilon}(\Omega)$ for some $\varepsilon>0$. By the compactness result in Proposition 4.1, we obtain that, up to a subsequence, $u_{n} \rightarrow u$ strongly in $W_{0}^{1, r}(\Omega)$ for all $r<p_{*}$ and $\left|\nabla u_{n}\right| \rightarrow|\nabla u|$ a.e. in $\Omega$. Hence by Vitali lemma we reach that $u_{n} \rightarrow u$ strongly in $W_{0}^{1, \alpha}(\Omega)$ with $\alpha$ previously chosen. Since $p<\alpha$, then

$$
\frac{\left|\nabla u_{n}\right|^{p}}{1+\frac{1}{n}\left|\nabla u_{n}\right|^{p}} \rightarrow|\nabla u|^{p} \text { strongly in } L^{1}(\Omega)
$$

Hence, $u$ is a solution to (34) with $u \in W_{0}^{1, \alpha}(\Omega)$.

Second case: $1<p \leqslant p_{-}(\lambda, s)$. We begin by proving that problem (16) has a supersolution in a small ball $B_{r}(0)$ that enjoys the same regularity properties as $w$.

Fix $p_{0} \in\left(p_{-}(\lambda, s), p_{+}(\lambda, s)\right)$ and consider $u_{p_{0}}$ the solution to problem (34) obtained in the first case with $f \equiv \frac{1}{|x|^{2 s}}$. Since $p_{0}>p$, then for all $\varepsilon>0$ there exists $C(\varepsilon)>0$ such that for all $\sigma \geqslant 0$, $\sigma^{p_{0}} \geqslant C(\varepsilon) \sigma^{p}-\varepsilon$. Thus

Therefore,

$$
\left|\nabla u_{p_{0}}\right|^{p_{0}} \geqslant C(\varepsilon)\left|\nabla u_{p_{0}}\right|^{p}-\varepsilon
$$

$$
(-\Delta)^{s} u_{p_{0}} \geqslant \lambda \frac{u_{p_{0}}}{|x|^{2 s}}+C(\varepsilon)\left|\nabla u_{p_{0}}\right|^{p}-\varepsilon+\frac{\mu}{|x|^{2 s}} \text { in } \Omega .
$$

Let fix $r>0$ small enough such that $\frac{\mu}{|x|^{2 s}}-\varepsilon \geqslant \frac{\mu_{0}}{|x|^{2 s}}$ in $B_{r}(0)$. Hence we conclude that $u_{p_{0}}$ satisfies

$$
(-\Delta)^{s} u_{p_{0}} \geqslant \lambda \frac{u_{p_{0}}}{|x|^{2 s}}+C(\varepsilon)\left|\nabla u_{p_{0}}\right|^{p}+\frac{\mu_{0}}{|x|^{2 s}} \text { in } B_{r}(0) .
$$

Setting $u_{p}=C(\varepsilon) u_{p_{0}}$, we reach that

$$
(-\Delta)^{s} u_{p} \geqslant \lambda \frac{u_{p}}{|x|^{2 s}}+\left|\nabla u_{p}\right|^{p}+\frac{\mu_{1}}{|x|^{2 s}} \text { in } B_{r}(0) .
$$

Thus $u_{p}$ is a supersolution to problem (34) with the same regularity properties as $w$. Hence the existence result follows using the same approach as in the first case.

Notice that if $u$ is a supersolution in $B_{r}(0)$, then for $x \in B_{R}(0)$ with $R>r$ and by setting $\hat{u}(x)=u\left(\frac{r}{R} x\right)$, then there exists a constant $C:=C(R, r, p)$ such that $\check{u}:=C \hat{u}$ is a supersolution to (34) in $B_{R}(0)$ with $\mu:=\hat{\mu}$.

Now we consider the case of general domain $\Omega$. Let $R>1$ be such that $\Omega \subset \subset B_{R}(0)$. It is clear that $\check{u}$ is a supersolution to (34) that has the same properties of $w$. Hence we conclude the proof of Theorem 4.5.

Corollary 4.6. Assume that $p<p_{+}(\lambda, s)$ and that $f \leqslant \frac{C}{|x|^{2 s}}$ with $f \geqslant 0$. Then there exists $\mu^{*}>0$ such that for all $\mu<\mu^{*}$, problem (34) has a positive solution u such that $u \in W_{0}^{1, \alpha}(\Omega)$ for all $\alpha<2 s$.

Remark 4.7. Under the extra assumption $p<p_{*}=\frac{N}{N-2 s+1}$, we are able to prove the existence of a solution for all $f \in L^{1}(\Omega)$ that satisfies a suitable integrability condition near the origin. More precisely, fixed $\lambda<\Lambda_{N, s}$, the problem

$$
\left\{\begin{aligned}
(-\Delta)^{s} v & =\lambda \frac{v}{|x|^{2 s}}+f & & \text { in } \Omega, \\
\phi & =0 & & \text { in } \mathbb{R}^{N} \backslash \Omega,
\end{aligned}\right.
$$


has a weak solution if and only if $\int_{\Omega} f|x|^{-\mu(\lambda)} d x<\infty$ (see [4]).

Moreover we have

$$
\int_{\Omega} v|x|^{-\mu(\lambda)} d x<\infty
$$

and

$\|v\|_{L^{1}\left(|x|^{-\mu(\lambda)} d x, \Omega\right)}+\left\|\frac{v}{|x|^{2 s}}\right\|_{L^{1}(\Omega)}+\|\nabla v \mid\|_{L^{\sigma}(\Omega)} \leqslant C(\Omega, \lambda)\|f\|_{L^{1}\left(|x|^{-\mu(\lambda)} d x, \Omega\right)}$, for all $\sigma<\frac{N}{N-2 s+1}$.

We refer to [4] for the proof.

Suppose now that $f \in L^{1}\left(|x|^{-\mu(\lambda)-a_{0}} d x, \Omega\right)$, hence there exists $\lambda_{1} \in\left(\lambda, \Lambda_{N, s}\right)$ such that $\mu\left(\lambda_{1}\right)=$ $\mu(\lambda)+a_{0}$. Define $\psi$ to be the unique solution to problem

$$
\left\{\begin{aligned}
(-\Delta)^{s} \psi & =\lambda_{1} \frac{\psi}{|x|^{2 s}}+1 & & \text { in } \Omega, \\
\psi & =0 & & \text { in } \mathbb{R}^{N} \backslash \Omega,
\end{aligned}\right.
$$

then $\psi \simeq|x|^{-\mu(\lambda)-a_{0}}$ near the origin. It is clear also that $\psi \in L^{\infty}\left(\Omega \backslash B_{r}(0)\right)$.

Using $\psi$ as a test function in problem (44), it holds that

$$
\left(\lambda_{1}-\lambda\right) \int_{\Omega} \frac{v \psi}{|x|^{2 s}} d x \leqslant \int_{\Omega} f \psi d x .
$$

Hence

$$
\int_{\Omega} \frac{v}{|x|^{2 s+\mu(\lambda)}} d x \leqslant C\left(\Omega \lambda, a_{0}\right)\|f\|_{L^{1}\left(|x|^{\left.-\mu(\lambda)-a_{0} d x, \Omega\right)}\right.} .
$$

The next proposition will be the key in order to show the existence of a solution to problem (34) under the above general hypothesis on $f$.

Proposition 4.8. Assume that $f \in L^{1}\left(|x|^{-\mu(\lambda)-a_{0}} d x, \Omega\right)$ for some $a_{0}>0$ and $v$ to be the unique weak solution to problem (44), then

$$
\|\nabla v\|_{L^{\alpha}\left(|x|^{-\mu(\lambda)} d x, \Omega\right)} \leqslant C\left(\Omega, \lambda, a_{0}\right)\|f\|_{L^{1}\left(|x|^{\left.-\mu(\lambda)-a_{0} d x, \Omega\right)}\right.} \text { for all } \alpha<\frac{N}{N-2 s+1} .
$$

Proof. Notice that

$$
\|\nabla v\|_{L^{\alpha}(\Omega)} \leqslant C\left(\Omega, \lambda, a_{0}\right)\|f\|_{L^{1}\left(|x|^{\left.-\mu(\lambda)-a_{0} d x, \Omega\right)}\right.} \text { for all } \alpha<\frac{N}{N-2 s+1} .
$$

Hence to prove the claim we have just to show that

$$
\int_{B_{r}(0)}|\nabla v|^{\alpha}|x|^{-\mu(\lambda)} d x \leqslant C\left(\Omega, \lambda, a_{0}\right)|| f \|_{L^{1}\left(|x|^{\left.-\mu(\lambda)-a_{0} d x, \Omega\right)}\right.} \text { for all } \alpha<\frac{N}{N-2 s+1} .
$$

We set $g(x):=\lambda \frac{v}{|x|^{2 s}}+\mu f$, then $v(x)=\int_{\Omega} \mathcal{G}_{s}(x, y) g(y) d y$. Hence

$$
|\nabla v(x)| \leqslant \int_{\Omega}\left|\nabla_{x} \mathcal{G}_{s}(x, y)\right| g(y) d y .
$$


Fix $1<\alpha<p_{*}=\frac{N}{N-2 s+1}$ and define $h(x, y)=\max \left\{\frac{1}{|x-y|}, \frac{1}{\delta(x)}\right\}$. Then,

$$
\begin{aligned}
|\nabla v(x)|^{\alpha} & \leqslant\left(\int_{\Omega}\left|\nabla_{x} \mathcal{G}_{s}(x, y)\right| g(y) d y\right)^{\alpha} \leqslant\left(\int_{\Omega} h(x, y) \mathcal{G}_{s}(x, y) g_{n}(y) d y\right)^{\alpha} \\
& \leqslant\left(\int_{\Omega}(h(x, y))^{\alpha} \mathcal{G}_{s}(x, y) g(y) d y\right)\left(\int_{\Omega} \mathcal{G}_{s}(x, y) g(y) d y\right)^{\alpha-1} \\
& \leqslant \int_{\Omega}\left(h^{\alpha}(x, y) \mathcal{G}_{s}(x, y) g(y) d y\right) v^{\alpha-1}(x) \\
& \leqslant \lambda \int_{\Omega}\left(h^{\alpha}(x, y) \mathcal{G}_{s}(x, y) \frac{v(y)}{|y|^{2 s}} d y\right) v^{\alpha-1}(x)+\mu \int_{\Omega}\left(h^{\alpha}(x, y) \mathcal{G}_{s}(x, y) f(y) d y\right) v^{\alpha-1}(x) .
\end{aligned}
$$

Thus

$$
\begin{aligned}
\int_{B_{r}(0)}|\nabla v|^{\alpha}|x|^{-\mu(\lambda)} d x & \leqslant \lambda \int_{\Omega} \frac{v(y)}{|y|^{2 s}}\left(\int_{B_{r}(0)} h^{\alpha}(x, y) \mathcal{G}_{s}(x, y) v^{\alpha-1}(x)|x|^{-\mu(\lambda)} d x\right) d y \\
& +\mu \int_{\Omega} f(y)\left(\int_{B_{r}(0)} h^{\alpha}(x, y) \mathcal{G}_{s}(x, y) v^{\alpha-1}(x)|x|^{-\mu(\lambda)} d x\right) d y \\
& \equiv J_{1}+J_{2} .
\end{aligned}
$$

Recall that $h(x, y)=\max \left\{\frac{1}{|x-y|}, \frac{1}{\delta(x)}\right\}$. then for all $x \in B_{r}(0) \subset \subset \Omega$, we have

$$
\frac{C_{1}\left(\Omega, B_{r}(0)\right)}{|x-y|} \leqslant h(x, y) \leqslant \frac{C_{2}\left(\Omega, B_{r}(0)\right)}{|x-y|} .
$$

Let us begin by estimating $J_{1}$. Recall that, by (47), we have

$$
\int_{\Omega} \frac{v(y)}{|y|^{2 s+\mu(\lambda)}} d y \leqslant C \int_{\Omega} \frac{f(y)}{|y|^{\mu(\lambda)+a_{0}}} d y .
$$

Therefore we obtain that

$$
\begin{aligned}
J_{1} & \leqslant C_{2} \int_{\Omega} \frac{v(y)}{|y|^{2 s}}\left(\int_{B_{r}(0)} \frac{v^{\alpha-1}(x) \mathcal{G}_{s}(x, y)}{|x|^{\mu(\lambda)}|x-y|^{\alpha}} d x\right) d y \\
& \leqslant C_{2} \int_{\Omega} \frac{v(y)}{|y|^{2 s}}\left(\int_{B_{r}(0)} \frac{v^{\alpha-1}(x)}{|x|^{\mu(\lambda)}|x-y|^{N-(2 s-\alpha)}} d x\right) d y \\
& \leqslant C \int_{\Omega} \frac{v(y)}{|y|^{2 s}}\left(\int_{B_{r}(0) \cap\left\{|x| \geqslant \frac{1}{2}|y|\right\}} \frac{v^{\alpha-1}(x)}{|x|^{\mu(\lambda)}|x-y|^{N-(2 s-\alpha)}} d x\right) d y \\
& +C \int_{\Omega} \frac{v(y)}{|y|^{2 s}}\left(\int_{B_{r}(0) \cap\left\{|x|<\frac{1}{2}|y|\right\}} \frac{v^{\alpha-1}(x)}{|x|^{\mu(\lambda)}|x-y|^{N-(2 s-\alpha)}} d x\right) d y \\
& \leqslant J_{11}+J_{12} .
\end{aligned}
$$


To estimate $J_{11}$, we have

$$
J_{11} \leqslant C \int_{\Omega} \frac{v(y)}{|y|^{2 s+\mu(\lambda)}}\left(\int_{B_{r}(0)} \frac{v^{\alpha-1}(x)}{|x-y|^{N-(2 s-\alpha)}} d x\right) d y .
$$

Recall that $v \in L^{\sigma}(\Omega)$ for all $\sigma<p_{2}=\frac{N}{N-2 s}$. Since $\alpha<p_{*}=\frac{N}{N-2 s+1}$. Let $\sigma_{0}<p_{2}$ and using Hölder inequality we obtain that

$$
\int_{B_{r}(0)} \frac{v^{\alpha-1}(x)}{|x-y|^{N-(2 s-\alpha)}} d x \leqslant\left(\int_{B_{r}(0)} v^{\sigma_{0}} d x\right)^{\frac{\alpha-1}{\sigma_{0}}}\left(\int_{B_{r}(0)} \frac{1}{|x-y|^{\frac{\left(N-(2 s-\alpha) \sigma_{0}\right.}{\sigma_{0}-(\alpha-1)}}} d x\right)^{\frac{\sigma_{0}-(\alpha-1)}{\sigma_{0}}} .
$$

Since $\alpha<p_{*}$, then we can chose $\sigma_{0}$ close to $p_{2}$ such that $\frac{(N-(2 s-\alpha)) \sigma_{0}}{\sigma_{0}-(\alpha-1)}<N$. Thus

$$
\int_{B_{r}(0)} \frac{1}{|x-y|^{\frac{(N-(2 s-\alpha)) \sigma_{0}}{\sigma_{0}-(\alpha-1)}}} d x \leqslant C(r, \Omega)
$$

and then

$$
J_{11} \leqslant C\left(\int_{B_{r}(0)} v^{\sigma_{0}} d x\right)^{\frac{\alpha-1}{\sigma_{0}}}\left(\int_{\Omega} \frac{v(y)}{|y|^{2 s+\mu(\lambda)}} d y\right) \leqslant C\left(\int_{\Omega} \frac{f(y)}{|y|^{\mu(\lambda)+a_{0}}} d y\right)^{\alpha} .
$$

We deal now with $J_{12}$. Notice that $\left\{|x| \leqslant \frac{1}{2}|y|\right\} \subset\left\{|x-y| \geqslant \frac{1}{2}|y|\right\}$. Thus

$$
J_{12} \leqslant C \int_{\Omega} \frac{v(y)}{|y|^{\mu(\lambda)+2 s}}\left(\int_{B_{r}(0)} \frac{v^{\alpha-1}(x)}{|x|^{\mu(\lambda)}|x-y|^{N-(2 s+\mu(\lambda)-\alpha)}} d x\right) d y .
$$

As in the estimate of $J_{11}$, setting $\theta=\frac{\sigma_{0}}{\sigma_{0}-(\alpha-1)}$, we have

$$
\begin{gathered}
\int_{B_{r}(0)} \frac{v^{\alpha-1}(x)}{|x|^{\mu(\lambda)}|x-y|^{N-(2 s+\mu(\lambda)-\alpha)}} d x \\
\leqslant\left(\int_{B_{r}(0)} v^{\sigma_{0}} d x\right)^{\frac{\alpha-1}{\sigma_{0}}}\left(\int_{B_{r}(0)} \frac{1}{|x|^{\mu(\lambda) \theta}|x-y|^{(N-(2 s+\mu(\lambda)-\alpha)) \theta}} d x\right)^{\frac{1}{\theta}} .
\end{gathered}
$$

Since $\mu(\lambda) \theta<N$ ( for $\sigma_{0}$ close to $p_{2}$ ), using again Hölder inequality, we obtain that

$$
\begin{gathered}
\int_{B_{r}(0)} \frac{1}{|x|^{\mu(\lambda) \theta}|x-y|^{(N-(2 s+\mu(\lambda)-\alpha)) \theta}} d x \leqslant \\
\left(\int_{B_{r}(0)} \frac{1}{|x|^{N-\varepsilon}} d x\right)^{\frac{\mu(\lambda) \theta}{N-\varepsilon}}\left(\int_{B_{r}(0)} \frac{1}{|x-y|^{\frac{(N-(2 s+\mu(\lambda)-\alpha) \theta(N-\varepsilon)}{N-\varepsilon-\mu(\lambda) \theta}}} d x\right)^{\frac{N-\varepsilon-\mu(\lambda) \theta}{N-\varepsilon}} .
\end{gathered}
$$

By a direct computation and using the fact that $\alpha<p_{*}$, we obtain that $\frac{(N-(2 s+\mu(\lambda)-\alpha)) \theta N}{N-\mu(\lambda) \theta}<N$. Hence we get the existence of $\varepsilon>0$ small such that $\frac{(N-(2 s+\mu(\lambda)-\alpha)) \theta(N-\varepsilon)}{N-\varepsilon-\mu(\lambda) \theta}<N$ and we conclude that

$$
J_{12} \leqslant C\left(\int_{\Omega} \frac{f(y)}{|y|^{\mu(\lambda)+a_{0}}} d y\right)^{\alpha} .
$$

As a consequence, we have

$$
J_{1} \leqslant C\left(\int_{\Omega} \frac{f(y)}{|y|^{\mu(\lambda)+a_{0}}} d y\right)^{\alpha}
$$


We deal now with $J_{2}$. We will use the same decomposition as in the estimate of $J_{1}$.

$$
\begin{aligned}
J_{2} & \leqslant C \int_{\Omega} f(y)\left(\int_{B_{r}(0)} \frac{v^{\alpha-1}(x)}{|x|^{\mu(\lambda)}|x-y|^{N-(2 s-\alpha)}} d x\right) d y \\
& \leqslant C \int_{\Omega} f(y)\left(\int_{B_{r}(0) \cap\left\{|x| \geqslant \frac{1}{2}|y|\right\}} \frac{v^{\alpha-1}(x)}{|x|^{\mu(\lambda)}|x-y|^{N-(2 s-\alpha)}} d x\right) d y \\
& +C_{3} \int_{\Omega} f(y)\left(\int_{B_{r}(0) \cap\left\{|x|<\frac{1}{2}|y|\right\}} \frac{v^{\alpha-1}(x)}{|x|^{\mu(\lambda)}|x-y|^{N-(2 s-\alpha)}} d x\right) d y \\
& \leqslant J_{21}+J_{22} .
\end{aligned}
$$

To estimate $J_{21}$, we have

$$
J_{21} \leqslant C_{3} \int_{\Omega} \frac{f(y)}{|y|^{\mu(\lambda)}}\left(\int_{B_{r}(0)} \frac{v^{\alpha-1}(x)}{|x-y|^{N-(2 s-\alpha)}} d x\right) d y .
$$

For the integral $\int_{B_{r}(0)} \frac{v^{\alpha-1}(x)}{|x-y|^{N-(2 s-\alpha)}} d x$, we use the same computations as in the estimate of $J_{11}$ (since we are with the same range of parameters), and then we conclude that

$$
\begin{aligned}
\int_{B_{r}(0)} \frac{v^{\alpha-1}(x)}{|x-y|^{N-(2 s-\alpha)}} d x & \leqslant\left(\int_{B_{r}(0)} v^{\sigma_{0}} d x\right)^{\frac{\alpha-1}{\sigma_{0}}}\left(\int_{B_{r}(0)} \frac{1}{|x-y|^{\frac{\left(N-(2 s-\alpha) \sigma_{0}\right.}{\sigma_{0}-(\alpha-1)}}} d x\right)^{\frac{\sigma_{0}-(\alpha-1)}{\sigma_{0}}} \\
& \leqslant C\left(\int_{B_{r}(0)} v^{\sigma_{0}} d x\right)^{\frac{\alpha-1}{\sigma_{0}}}
\end{aligned}
$$

Thus,

$$
J_{21} \leqslant C\left(\int_{B_{r}(0)} v^{\sigma_{0}} d x\right)^{\frac{\alpha-1}{\sigma_{0}}}\left(\int_{\Omega} \frac{f(y)}{|y|^{\mu(\lambda)}} d y\right) \leqslant C\left(\int_{\Omega} \frac{f(y)}{|y|^{\mu(\lambda)}} d y\right)^{\alpha} .
$$

To analyze $J_{22}$ we use also the fact that $\left\{|x| \leqslant \frac{1}{2}|y|\right\} \subset\left\{|x-y| \geqslant \frac{1}{2}|y|\right\}$. Then

$$
J_{22} \leqslant C \int_{\Omega} \frac{f(y)}{|y|^{\mu(\lambda)}}\left(\int_{B_{r}(0)} \frac{v^{\alpha-1}(x)}{|x|^{\mu(\lambda)}|x-y|^{N-(2 s+\mu(\lambda)-\alpha)}} d x\right) d y .
$$

As in the estimate of $J_{12}$, it holds that

$$
\begin{gathered}
\int_{B_{r}(0)} \frac{v^{\alpha-1}(x)}{|x|^{\mu(\lambda)}|x-y|^{N-(2 s+\mu(\lambda)-\alpha)}} d x \\
\leqslant\left(\int_{B_{r}(0)} v^{\sigma_{0}} d x\right)^{\frac{\alpha-1}{\sigma_{0}}}\left(\int_{B_{r}(0)} \frac{1}{|x|^{\mu(\lambda) \theta}|x-y|^{(N-(2 s+\mu(\lambda)-\alpha)) \theta}} d x\right)^{\frac{1}{\theta}} \leqslant C\left(\int_{B_{r}(0)} v^{\sigma_{0}} d x\right)^{\frac{\alpha-1}{\sigma_{0}}} .
\end{gathered}
$$

Therefore, we obtain

$$
J_{22} \leqslant C\left(\int_{\Omega} \frac{f(y)}{|y|^{\mu(\lambda)}} d y\right)^{\alpha}
$$


Hence,

$$
J_{2} \leqslant C\left(\int_{\Omega} \frac{f(y)}{|y|^{\mu(\lambda)}} d y\right)^{\alpha}
$$

From (51) and (54) it holds that

$$
\int_{B_{r}(0)}|\nabla v|^{\alpha}|x|^{-\mu(\lambda)} d x \leqslant C\left(\int_{\Omega} \frac{f(y)}{|y|^{\mu(\lambda)}} d y\right)^{\alpha},
$$

and then result follows.

With all the above machinery, we are able to show the next existence result.

Theorem 4.9. Assume that $1<p<p_{*}$ and let $f \in L^{1}(\Omega)$ be a nonnegative function such that $\int_{\Omega} f|x|^{-\mu(\lambda)-a_{0}} d x<\infty$ for some $a_{0}>0$. Then, there exists $\mu^{*}>0$ such that if $\mu<\mu^{*}$, then problem (34) has a solution $u$ such that $u \in W_{0}^{1, \sigma}(\Omega)$ for all $\sigma<\frac{N}{N-2 s+1}$, moveover $\int_{\Omega}|\nabla u|^{p}|x|^{-\mu(\lambda)} d x<\infty$.

Proof. We follow again the arguments used in [6]. Fix $p<p_{*}$ and let $f \in L^{1}(\Omega)$ be a nonnegative function with $\int_{\Omega} f|x|^{-\mu(\lambda)-a_{0}} d x<\infty$.

Fix $1<p<r<p_{*}$. Then, we can chose $\mu^{*}>0$ such that for some $l>0$, we have

$$
C_{0}\left(l+\mu^{*}|| f \|_{L^{1}\left(|x|^{-\mu(\lambda)} d x, \Omega\right)}\right)=l^{\frac{1}{p}},
$$

where $C_{0}$ is a positive constant depending only on $\Omega, \lambda$ and $C(\Omega, \lambda)$ given in (45).

Let $\mu<\mu^{*}$ be fixed and define the set

$$
\left.E=\left\{v \in W_{0}^{1,1}(\Omega): v \in W_{0}^{1, r}\left(|x|^{-\mu(\lambda)} d x, \Omega\right) \text { and }\|\nabla v\|_{L^{r}(|x|-\mu(\lambda)} d x, \Omega\right) \leqslant l^{\frac{1}{p}}\right\},
$$

where $p<r<p_{*}$. It is clear that $E$ is a closed convex set of $W_{0}^{1,1}(\Omega)$. Consider the operator

$$
\begin{aligned}
T: E & \rightarrow W_{0}^{1,1}(\Omega) \\
v & \rightarrow T(v)=u
\end{aligned}
$$

where $u$ is the unique solution to problem

$$
\left\{\begin{aligned}
(-\Delta)^{s} u & =\lambda \frac{u}{|x|^{2 s}}+|\nabla v|^{q}+\mu f & & \text { in } \Omega, \\
u & =0 & & \text { in } \mathbb{R}^{N} \backslash \Omega, \\
u & >0 & & \text { in } \Omega .
\end{aligned}\right.
$$

Taking into consideration the definition of $E$, it holds that $|\nabla v|^{q}+\mu f \in L^{1}\left(|x|^{-\mu(\lambda)} d x, \Omega\right)$. Hence the existence and the uniqueness of $u$ follows using the result of [4] with $u \in W_{0}^{1, \sigma}(\Omega)$ for all $\sigma<\frac{N}{N-2 s+1}$. Thus $T$ is well defined.

We claim that $T(E) \subset E$. Since $r>p$, then using Hölder inequality we get the existence of $\hat{a}_{0}>0$ such that

$$
\int_{\Omega}|\nabla v|^{p}|x|^{-\mu(\lambda)-\hat{a}_{0}} d x \leqslant C(\Omega)\left(\int_{\Omega}|\nabla v|^{r}|x|^{-\mu(\lambda)} d x\right)^{\frac{p}{r}}<\infty .
$$

Setting $\bar{a}_{0}=\min \left\{a_{0}, \hat{a}_{0}\right\}$, it holds that $|\nabla v|^{p}+\mu f \in L^{1}\left(|x|^{-\mu(\lambda)-\bar{a}} d x, \Omega\right)$. Thus by Proposition 4.8, we reach

$$
\left(\int_{\Omega}|\nabla u|^{\sigma}|x|^{-\mu(\lambda)} d x\right)^{\frac{1}{\sigma}} \leqslant C(N, p, \bar{a})\left\||\nabla v|^{p}+\mu f\right\|_{L^{1}\left(|x|^{-\mu(\lambda)-\bar{a}} d x, \Omega\right)} .
$$


Since $v \in E$, we conclude that

$$
\begin{aligned}
\left(\int_{\Omega}|\nabla u|^{\sigma}|x|^{-\mu(\lambda)} d x\right)^{\frac{1}{\sigma}} & \leqslant C(N, p, \bar{a})\left(\left(\int_{\Omega}|\nabla v|^{r}|x|^{-\mu(\lambda)} d x\right)^{\frac{p}{r}}+\mu\|f\|_{L^{1}\left(|x|^{\left.-\mu(\lambda)-a_{0} d x, \Omega\right)}\right.}\right) \\
& \leqslant C\left(l+\mu^{*}|| f \|_{L^{1}\left(|x|^{\left.-\mu(\lambda)-a_{0} d x, \Omega\right)}\right.}\right) \leqslant l .
\end{aligned}
$$

Choosing $\sigma=r$, it holds that $u \in E$.

The continuity and the compactness of $T$ follow using closely the same arguments as in [6].

As a conclusion and using the Schauder Fixed Point Theorem as in [6], there exists $u \in E$ such that $T(u)=u, u \in W_{0}^{1, p}\left(|x|^{-\mu(\lambda)} d x, \Omega\right)$ and, therefore, $u$ solves (34).

\section{Existence Under the PRESEnCE of a ZERo order term VANiShing AT INFINITy.}

In this section we consider the problem

$$
\left\{\begin{aligned}
(-\Delta)^{s} u & =\lambda \frac{u}{|x|^{2 s}}+\frac{|\nabla u|^{p}}{(1+u)^{\alpha}}+c f & & \text { in } \Omega, \\
u & >0 & & \text { in } \Omega, \\
u & =0 & & \text { in }\left(\mathbb{R}^{N} \backslash \Omega\right),
\end{aligned}\right.
$$

where $\alpha>0$ and $p<2 s$. The main objective of this section is to get a relation between $\alpha$ and $p$ in order to get the existence of a solution for some $p>p_{+}(\lambda, s)$.

The local case was treated in [3] where the term $\frac{1}{(1+u)^{\alpha}}$ is replaced by $\frac{1}{u^{\alpha}}$. The problem in this case is strongly related to the porus medium equation with Hardy potential. Existence result is obtained under the condition that $\frac{(\mu(\lambda)+1)(p-1)-1}{\mu(\lambda)}<\alpha<p$, where $\mu(\lambda)$ is defined in (14). The arguments used in [3] are based on the choose of suitable text functions and the connection between the laplacian operator and the gradient term through the integration by parts formula.

This approach fails in the case of the fractional Laplacian due to the nonlocal nature of the operator and the like of a direct relation between the fractional Laplacian and the gradient term. To overcome these difficulties, we will use monotony argument and the representation formula.

The main existence result of this section is the following.

Theorem 5.1. Assume that $\alpha>2 s-1$. Suppose que $0 \supsetneqq f \leqslant \frac{1}{|x|^{\beta}}$ where $\mu(\lambda)<\beta<\bar{\mu}(\lambda)$ and $\beta$ is close to $\mu(\lambda)$. Then there exists $c^{*}>0$ such that if $c<c^{*}$, then problem (57) has a solution in the sense of Definition 2.2 with $\frac{u}{|x|^{2 s}}+\frac{|\nabla u|^{p}}{(1+u)^{\alpha}} \in L^{1}(\Omega)$.

Before starting with the proof of the previous Theorem, we state the next comparison principle.

Proposition 5.2. Assume that $H: \mathbb{R}^{N} \rightarrow \mathbb{R}^{N}$ is a bounded function satisfying

$$
\left|H\left(\xi_{1}\right)-H\left(\xi_{2}\right)\right| \leqslant C\left|\xi_{1}-\xi_{2}\right|, \quad \forall \xi_{1}, \xi_{2} \in \mathbb{R}^{N}, \quad C>0 .
$$

Consider $w_{1}, w_{2}$ positive functions such that $w_{1}, w_{2} \in W_{0}^{1, r}(\Omega), 1<r<\frac{N}{N-2 s+1}$, satisfying

$$
\left\{\begin{aligned}
(-\Delta)^{s} w_{1} & \leqslant \frac{1}{\left(w_{1}+1\right)^{\alpha}} H\left(\nabla w_{1}\right)+g, & & \text { in } \Omega, \\
w_{1} & =0 & & \text { on } \partial \Omega,
\end{aligned}\right.
$$


and

$$
\left\{\begin{aligned}
(-\Delta)^{s} w_{2} & \geqslant \frac{1}{\left(w_{2}+1\right)^{\alpha}} H\left(\nabla w_{2}\right)+g, & & \text { in } \Omega, \\
w_{2} & =0 & & \text { on } \partial \Omega,
\end{aligned}\right.
$$

where $\alpha>0$ and $g \in L^{1}(\Omega)$. Then, $w_{2} \geqslant w_{1}$ in $\Omega$.

Proof. Define $w=w_{1}-w_{2}$, then $w \in W_{0}^{1, r}(\Omega)$ with $1<r<\frac{N}{N-2 s+1}$. We have just to show that $w^{+}=0$.

Using (58) and (59), it follows that

$$
(-\Delta)^{s} w \leqslant \frac{1}{\left(w_{1}+1\right)^{\alpha}}\left(H\left(\nabla w_{1}\right)-H\left(\nabla w_{2}\right)\right)+\left(\frac{1}{\left(w_{1}+1\right)^{\alpha}}-\frac{1}{\left(w_{2}+1\right)^{\alpha}}\right) H\left(\nabla w_{2}\right),
$$

then,

$$
(-\Delta)^{s} w \leqslant C \frac{1}{\left(w_{1}+1\right)^{\alpha}}|\nabla w|+\left(\frac{1}{\left(w_{1}+1\right)^{\alpha}}-\frac{1}{\left(w_{2}+1\right)^{\alpha}}\right) H\left(\nabla w_{2}\right) .
$$

Since the second member in the previous inequality is bounded in $L^{1}(\Omega)$, then using Kato's inequality, we get

$$
(-\Delta)^{s} w_{+} \leqslant C\left|\nabla w_{+}\right|, \quad w_{+}=\max \{\mathrm{w}, 0\} \in \mathrm{W}_{0}^{1, \mathrm{r}}(\Omega) .
$$

Therefore, by using the maximum principle obtained in Theorem 3.1 of [6], we reach that $w_{+} \equiv 0$. Hence we conclude.

Now, let $\beta>0$ be such that $\mu(\lambda)<\beta<\bar{\mu}(\lambda), \beta$ is close to $\mu(\lambda)$ in such a way that $|x|^{-\beta-2 s} \in L^{2}(\Omega)$, hence $(-\Delta)^{s}|x|^{-\beta} \in L^{2}(\Omega)$.

Define $v(x)=\frac{A}{|x|^{\beta}}$, since $\alpha>2 s-1$, then $\frac{\mu(\lambda)(\alpha+1)+2 s}{\mu(\lambda)+1}>2 s>p$. Hence we get the existence of $\beta>0$ such that $\mu(\lambda)<\beta<\bar{\mu}(\lambda), \beta$ closed to $\mu(\lambda)$ and $\frac{\beta(\alpha+1)+2 s}{\beta+1}>2 s>p$.

Fix $\beta$ as above, then we get the existence of $A>0$ and $c^{*}>0$ such that if $f(x) \leqslant \frac{1}{|x|^{\beta+2 s}}$ and $c<c^{*}$,

$$
(-\Delta)^{s} v \geqslant \lambda \frac{v}{|x|^{2 s}}+\frac{|\nabla v|^{p}}{v^{\alpha}}+c f \text { in } \Omega .
$$

Hence $v$ is a supersolution to problem (35).

\section{Proof of Theorem 5.1.}

We will use a monotony argument.

Define now $u_{n}$ to be the minimal solution to the approximating problem

$$
\left\{\begin{array}{rlrl}
(-\Delta)^{s} u_{n} & =\lambda \frac{u_{n}}{1+\frac{1}{n} u_{n}} \frac{1}{|x|^{2 s}}+\frac{\left|\nabla u_{n}\right|^{p}}{\left(1+\frac{1}{n}\left|\nabla u_{n}\right|^{p}\right)\left(1+u_{n}\right)^{\alpha}}+c f & & \text { in } \Omega, \\
u_{n} & >0 & \text { in } \Omega, \\
u_{n} & =0 & & \text { in }\left(\mathbb{R}^{N} \backslash \Omega\right) .
\end{array}\right.
$$

Since $v$ is a supersolution to problem (60), then using the comparison principle in Proposition 5.2 it holds that the sequence $\left\{u_{n}\right\}_{n}$ is increasing in $n$ and $u_{n} \leqslant v$ for all $n$. Hence we get the existence of a measurable function $u$ such that $u_{n} \nearrow u$ strongly in $L^{\theta}(\Omega)$ for all $\theta<\frac{N}{\beta}$ and $u \leqslant v$ in $\mathbb{R}^{N}$.

To simplify the notation, we set

$$
g_{1 n}(x):=\frac{\left|\nabla u_{n}\right|^{p}}{\left(1+\frac{1}{n}\left|\nabla u_{n}\right|^{p}\right)\left(1+u_{n}\right)^{\alpha}}, g_{2 n}(x):=\lambda \frac{u_{n}}{1+\frac{1}{n} u_{n}} \frac{1}{|x|^{2 s}}+c f,
$$


and $g_{n}=g_{n 1}+g_{n 2}$. Notice that $g_{2 n}(x) \leqslant \frac{C}{|x|^{\beta+2 s}}$ in $\Omega$. It is not difficult to show that $\int_{\Omega}\left(g_{1 n}+\right.$ $\left.g_{2 n}\right) \delta^{s} d x \leqslant C$ for all $n$.

We claim that $\left\|g_{n}\right\|_{L^{1}(\Omega)} \leqslant C$ for all $n$. It is clear that $\left\|g_{2 n}\right\|_{L^{1}(\Omega)} \leqslant C$ for all $n$.

Using the definition of $u_{n}$, we have

$$
u_{n}(x)=\int_{\Omega} \mathcal{G}_{s}(x, y) g_{n}(y) d y \quad \text { and then }\left|\nabla u_{n}(x)\right| \leqslant \int_{\Omega}\left|\nabla_{x} \mathcal{G}_{s}(x, y)\right| g_{n}(y) d y .
$$

Hence, for $p<\sigma<2 s$, to be chosen later, it follows that

$$
\left|\nabla u_{n}(x)\right|^{\sigma} \leqslant\left(\int_{\Omega}\left|\nabla_{x} \mathcal{G}_{s}(x, y)\right| g_{n}(y) d y\right)^{\sigma} \leqslant\left(\int_{\Omega} \frac{\left|\nabla_{x} \mathcal{G}_{s}(x, y)\right|}{\mathcal{G}_{s}(x, y)} \mathcal{G}_{s}(x, y) g_{n}(y) d y\right)^{\sigma} .
$$

Recall that $h(x, y)=\max \left\{\frac{1}{|x-y|}, \frac{1}{\delta(x)}\right\}$, form (41), it holds that

$$
\begin{aligned}
\left|\nabla u_{n}(x)\right|^{\sigma} & \leqslant\left(\int_{\Omega}(h(x, y))^{\alpha} \mathcal{G}_{s}(x, y) g_{n}(y) d y\right)\left(\int_{\Omega} \mathcal{G}_{s}(x, y) g_{n}(y) d y\right)^{\sigma-1} \\
& \leqslant\left(\int_{\Omega}\left(h^{\sigma}(x, y) \mathcal{G}_{s}(x, y) g_{n}(y) d y\right) u_{n}^{\sigma-1}(x) .\right.
\end{aligned}
$$

Thus

$$
\begin{gathered}
\frac{\left|\nabla u_{n}(x)\right|^{\sigma}}{\left(1+u_{n}\right)^{\sigma-1}} \leqslant \int_{\Omega}\left(h^{\sigma}(x, y) \mathcal{G}_{s}(x, y) g_{n}(y) d y\right) \frac{u_{n}^{\sigma-1}(x)}{\left(1+u_{n}(x)\right)^{\sigma-1}} \\
\leqslant \int_{\Omega}\left(h^{\sigma}(x, y) \mathcal{G}_{s}(x, y) g_{1 n}(y) d y\right) \frac{u_{n}^{\sigma-1}(x)}{\left(1+u_{n}(x)\right)^{\sigma-1}}+\int_{\Omega}\left(h^{\sigma}(x, y) \mathcal{G}_{s}(x, y) g_{2 n}(y) d y\right) \frac{u_{n}^{\sigma-1}(x)}{\left(1+u_{n}(x)\right)^{\sigma-1}} .
\end{gathered}
$$

By integrating in $x$, we get

$$
\begin{aligned}
\int_{\Omega} \frac{\left|\nabla u_{n}(x)\right|^{\sigma}}{\left(1+u_{n}\right)^{\sigma-1}} d x & \leqslant \int_{\Omega} g_{1 n}(y)\left(\int_{\Omega} h^{\sigma}(x, y) \mathcal{G}_{s}(x, y) \frac{u_{n}^{\sigma-1}(x)}{\left(1+u_{n}(x)\right)^{\sigma-1}} d x\right) d y \\
& +\lambda \int_{\Omega} g_{2 n}(y)\left(\int_{\Omega} h^{\sigma}(x, y) \mathcal{G}_{s}(x, y) \frac{u_{n}^{\sigma-1}(x)}{\left(1+u_{n}(x)\right)^{\sigma-1}} d x\right) d y \equiv J_{1}+J_{2} .
\end{aligned}
$$

Let us begin by estimating $J_{1}$.

$$
J_{1} \leqslant \int_{\Omega} \frac{\left|\nabla u_{n}(y)\right|^{p}}{\left(1+u_{n}(y)\right)^{\alpha}}\left(\int_{\Omega} \frac{\mathcal{G}_{s}(x, y)}{|x-y|^{\sigma}} d x+\int_{\Omega} \frac{\mathcal{G}_{s}(x, y)}{\delta^{\sigma}(x)} d x\right) d y .
$$

It is clear that

$$
\frac{\mathcal{G}_{s}(x, y)}{|x-y|^{\sigma}} \leqslant \frac{C(N, s)}{|x-y|^{N-2 s+\sigma}} .
$$

Thus using the fact that $\sigma<2 s$, it holds that

$$
\int_{\Omega} \frac{\mathcal{G}_{s}(x, y)}{|x-y|^{\sigma}} d x \leqslant C(\Omega, N, s)<\infty .
$$


Define now $\psi(y):=\int_{\Omega} \frac{\mathcal{G}_{s}(x, y)}{\delta^{\sigma}(x)} d x$, then

$$
\left\{\begin{aligned}
(-\Delta)^{s} \psi & =\frac{1}{\delta^{\sigma}(x)} & & \text { in } \Omega, \\
\psi & =0 & & \text { in }\left(\mathbb{R}^{N} \backslash \Omega\right) .
\end{aligned}\right.
$$

Using Theorem 1.2 in [9], we obtain that $\psi \simeq \delta^{2 s-\sigma}$. Therefore combining the above estimates, we reach that

$$
J_{1} \leqslant C(\Omega, N, s) \int_{\Omega} \frac{\left|\nabla u_{n}(y)\right|^{p}}{\left(1+u_{n}(y)\right)^{\alpha}} d y .
$$

We deal now with $J_{2}$. Recall that $g_{2 n}(y) \leqslant \frac{C}{|y|^{\beta+2 s}}$, thus

$$
J_{2} \leqslant \int_{\Omega} \frac{C}{|y|^{\beta+2 s}}\left(\int_{\Omega} \frac{\mathcal{G}_{s}(x, y)}{|x-y|^{\sigma}} d x\right) d y+\int_{\Omega} \frac{C}{|y|^{\beta+2 s}} \psi(y) d y .
$$

Hence, as in the computations of $J_{1}$,

$$
J_{2} \leqslant C \int_{\Omega} \frac{d y}{|y|^{\beta+2 s}} d y=C(N, s, \beta, \Omega)<\infty .
$$

Thus, we conclude that

$$
\int_{\Omega} \frac{\left|\nabla u_{n}(x)\right|^{\sigma}}{\left(1+u_{n}\right)^{\sigma-1}} d x \leqslant C_{1} \int_{\Omega} \frac{\left|\nabla u_{n}(x)\right|^{p}}{\left(1+u_{n}(x)\right)^{\alpha}} d x+C_{3} .
$$

Now, using Young inequality, it holds that

$$
\int_{\Omega} \frac{\left|\nabla u_{n}(x)\right|^{\sigma}}{\left(1+u_{n}\right)^{\sigma-1}} d x \leqslant \varepsilon \int_{\Omega} \frac{\left|\nabla u_{n}(x)\right|^{\sigma}}{\left(1+u_{n}\right)^{\sigma-1}} d x+C(\varepsilon) \int_{\Omega} \frac{1}{\left(1+u_{n}\right)^{\frac{\alpha \sigma-p(\sigma-1)}{\sigma-p}}} d x .
$$

Recall that $\sigma \in(p, 2 s)$, since $0<p-(2 s-1)<1$, then $p<\frac{p}{p-(2 s-1)}$. Thus we can chose $\sigma$ such that $p<\sigma<\max \left\{2 s, \frac{p}{p-(2 s-1)}\right\}$.

Hence $\alpha \sigma-p(\sigma-1) \geqslant 0$ and then $\int_{\Omega} \frac{1}{\left(1+u_{n}\right)^{\frac{\alpha \sigma-p(\sigma-1)}{\sigma-p}}} d x \leqslant|\Omega|$.

As a conclusion and choosing $\varepsilon$ small enough, we obtain that

$$
\int_{\Omega} \frac{\left|\nabla u_{n}\right|^{\sigma}}{\left(1+u_{n}\right)^{\sigma-1}} d x+\int_{\Omega} \frac{\left|\nabla u_{n}\right|^{p}}{\left(1+u_{n}\right)^{\alpha}} d x \leqslant C \text { for all } n .
$$

Hence $\left\|g_{2 n}\right\|_{L^{1}(\Omega)} \leqslant C$ for all $n$ and the claim follows.

By the compactness result in Theorem 4.1, we get the existence of $u \in W_{0}^{1, q}(\Omega)$, for all $q<\frac{N}{N-2 s+1}$, such that up to a subsequence, $u_{n} \rightarrow u$ strongly in $W_{0}^{1, \theta}(\Omega)$ for all $\theta<\frac{N}{N-2 s+1}$ and $\left|\nabla u_{n}\right| \rightarrow|\nabla u|$ a.e. in $\Omega$. Using Fatou's Lemma we reach that

$$
\int_{\Omega} \frac{|\nabla u|^{\sigma}}{(1+u)^{\sigma-1}} d x+\int_{\Omega} \frac{|\nabla u|^{p}}{(1+u)^{\alpha}} d x \leqslant C .
$$


Now, since $p<2 s$, then going back to estimate (61), choosing $\sigma \in(p, 2 s)$ and using Vitali lemma, we can prove that

$$
\frac{\left|\nabla u_{n}\right|^{p}}{\left(1+u_{n}\right)^{\alpha}} \rightarrow \frac{|\nabla u|^{p}}{(1+u)^{\alpha}} \text { strongly in } L^{1}(\Omega) .
$$

Thus $u$ is a solution to problem (57) with $u \in W_{0}^{1, \theta}(\Omega)$ for all $\theta<\frac{N}{N-2 s+1}$.

Remarks 5.3. Under additional hypothesis on $\alpha$, we can show the existence of a solution to the problem (57) where the term $\frac{1}{(1+u)^{\alpha}}$ is replaced by $\frac{1}{u^{\alpha}}$. More precisely, assume that $2 s-1<\alpha<$ $p+1-\frac{p}{s}$ (this is possible using the fact that $s>\frac{1}{2}$ and $1<p<2 s$ ). Now, we consider $u_{n}$ to be the minimal solution to the problem

$$
\left\{\begin{aligned}
(-\Delta)^{s} u_{n} & =\lambda \frac{u_{n}}{1+\frac{1}{n} u_{n}} \frac{1}{|x|^{2 s}}+\frac{\left|\nabla u_{n}\right|^{p}}{\left(1+\frac{1}{n}\left|\nabla u_{n}\right|^{p}\right)\left(\frac{1}{n}+u_{n}\right)^{\alpha}}+c f & & \text { in } \Omega, \\
u_{n} & >0 & & \text { in } \Omega, \\
u_{n} & =0 & & \text { in }\left(\mathbb{R}^{N} \backslash \Omega\right) .
\end{aligned}\right.
$$

As above, $v$ is a supersolution to (62) and then the increasing sequence $\left\{u_{n}\right\}_{n}$ satisfies $u_{n} \leqslant v$, for all $n$. It is clear that the only point that we have to prove is the fact that

$$
\left\|\frac{\left|\nabla u_{n}\right|^{p}}{\left(1+\frac{1}{n}\left|\nabla u_{n}\right|^{p}\right)\left(\frac{1}{n}+u_{n}\right)^{\alpha}}\right\|_{L^{1}(\Omega)} \leqslant C, \text { for all } n .
$$

Repeating the same computation as above we arrive to

$$
\int_{\Omega} \frac{\left|\nabla u_{n}(x)\right|^{\sigma}}{u_{n}^{\sigma-1}} d x \leqslant C_{1} \int_{\Omega} \frac{\left|\nabla u_{n}(x)\right|^{p}}{\left(\frac{1}{n}+u_{n}(x)\right)^{\alpha}} d x+C_{3} .
$$

Then by Young inequality,

$$
(1-\varepsilon) \int_{\Omega} \frac{\left|\nabla u_{n}(x)\right|^{\sigma}}{u_{n}^{\sigma-1}} d x \leqslant C(\varepsilon) \int_{\Omega} \frac{1}{u_{n}^{\frac{\alpha \sigma-p(\sigma-1)}{\sigma-p}}} d x+C_{3} .
$$

Since $2 s-1<\alpha<p+1-\frac{p}{s}$, then $p<\frac{p}{p-\alpha}$, hence choosing $\sigma$ such that

$$
\max \left\{p, \frac{p}{p+1-\alpha}\right\}<\sigma<\frac{p}{p-\alpha}<2 s,
$$

it holds that $\alpha \sigma-p(\sigma-1)>0$. Now, using the fact that the sequence $\left\{u_{n}\right\}_{n}$ is increasing in $n$ and since $f \supsetneqq 0$, then $u_{n} \geqslant u_{1} \geqslant C \delta^{s}$ for some universal constant and then

$$
\int_{\Omega} \frac{1}{u_{n}^{\frac{\alpha \sigma-p(\sigma-1)}{\sigma-p}}} d x \leqslant C \int_{\Omega} \frac{1}{\delta^{s \frac{\alpha \sigma-p(\sigma-1)}{\sigma-p}}} d x .
$$

Since $s \frac{\alpha \sigma-p(\sigma-1)}{\sigma-p}<1$, then $\int_{\Omega} \frac{1}{\delta^{s} \frac{\alpha \sigma-p(\sigma-1)}{\sigma-p}} d x<\infty$ and hence we conclude.

In a forthcoming work, we will analyze the general case without using monotony arguments and under general integrability assumptions on $f$. 


\section{REFERENCES}

[1] B. Abdellaoui, A. Attar, R. Bentifour, On the fractional p-laplacian equations with weights and general datum. Adv. Nonlinear Anal. 8 (2019), no. 1, 144-174.

[2] B. Abdellaoui, A. J. Fernández, Nonlinear fractional Laplacian problems with nonlocal gradient terms. Proceedings of the Royal Society of Edinburgh, (2020), 1-37. DOI:10.1017/prm.2019.60.

[3] B. Abdellaoui, D. Giachetti, I. Peral, M. Walias, Elliptic problems with nonlinear terms depending on the gradient and singular on the boundary: interaction with a Hardy-Leray potential. Discrete Contin. Dyn. Syst. 34 (2014), no. 5, 1747-1774.

[4] B. Abdellaoui, M. Medina, I. Peral and A. Primo, The effect of the Hardy potential in some Calderón-Zygmund properties for the fractional Laplacian. J. Differential Equations, 260 (2016) 8160-8206.

[5] B. Abdellaoui, I.Peral, The Equation $-\Delta u-\lambda \frac{u}{|x|^{2}}=|\nabla u|^{p}+c f(x)$, the optimal power. Ann. Scuola Norm. Sup. Pisa, (5) Vol VI, 2007, 159-183.

[6] B. Abdellaoui, I. Peral, Towards a deterministic KPZ equation with fractional diffusion: the stationary problem. Nonlinearity 31, 2018, 1260-1298.

[7] B. Abdellaoui, I. Peral, Towards a deterministic KPZ equation with fractional diffusion: the stationary problem, arXiv:1609.04561v3 [math.AP] 2 Oct 2019.

[8] N.E. AlaA, M. PIERRE, Weak solutions of some quasilinear elliptic equations with data measures. SIAM J. Math. Anal., 24, (1993), 23-35.

[9] Adimurthi, J. Giacomoni, S. Santra, Positive solutions to a fractional equation with singular nonlinearity. J. Differential Equations 265 (2018), no. 4, 1191-1226.

[10] W. Beckner, Pitt's inequality and the uncertainty principle. Proceedings of the American Mathematical Society, 123 (1995), no. 6, 1897-1905.

[11] K. Bogdan, T. Kulczycki, A. Nowak, Gradient estimates for harmonic and q-harmonic funcitons of Symmetric stable processes. Illinois J. Math. 46 (2002) no 2, 541-556.

[12] K. Bogdan, T. Kulczycki, M. Kwaśnicki, Estimates and structure of a-harmonic functions. Probab. Theory Relat. Fields, 140 (2008), 345-381.

[13] K. Bogdan, T. Jakubowski, Estimates of the Green Function for the Fractional Laplacian Perturbed by Gradient. Potential Anal 36 (2012), 455-481.

[14] H. Brezis, L. Dupaigne, A. Tesei, On a semilinear elliptic equation with inverse square potential. Selecta Math. (N.S.) 11 (2005), no. 1, 1-7.

[15] J. Dávila, L. Dupaigne, M. Montenegro, The extremal solution of a boundary reaction problem. Commun. Pure Appl. Anal. 7 (2008), no. 4, 795-817.

[16] H. Chen, L. Veron, Semilinear fractional elliptic equations involving measures. J. Differential Equations 257 (2014) 1457-1486.

[17] H. Chen, L. Veron, Semilinear fractional elliptic equations with gradient nonlinearity involving measures. Journal of Functional Analysis 266 (2014) 5467-5492.

[18] E. Di Nezza, G. Palatucci, E. Valdinoci, Hitchhiker's guide to the fractional Sobolev spaces. Bull. Sci. math. 136 (2012), no. 5, 521-573.

[19] R. L. Frank, E. H. Lieb, R. Seiringer, Hardy-Lieb-Thirring inequalities for fractional Schrödinger operators. J. Amer. Math. Soc., 21 (2008), 925-950.

[20] F. Ferrari, I. Verbitsky, Radial fractional Laplace operators and Hessian inequalities. J. Differential Equations 253 (2012), no. 1, 244-272.

[21] G. Karch, W.A. Woyczyński, Fractal Hamilton-Jacobi-KPZ equations. Trans. Amer. Math. Soc. 360 (2008), no. $5,2423-2442$.

[22] M. Kassmann, A priori estimates for integro-differential operators with measurable kernels. Calc. Var., 34 (2009), $1-21$.

[23] M. Kardar, G. Parisi, Y.C. Zhang, Dynamic scaling of growing interfaces. Phys. Rev. Lett. 56, (1986), 889-892.

[24] M. Kassmann, A priori estimates for integro-differential operators with measurable kernels. Calc. Var., 34 (2009), $1-21$.

[25] I. W. Herbst, Spectral theory of the operator $\left(p^{2}+m^{2}\right)^{1 / 2}-Z e^{2} / r$. Commun. math. Phys., 53 (1977), $285-294$.

[26] T. Leonori, I. Peral, A. Primo, F. Soria, Basic estimates for solution of elliptic and parabolic equations for a class of nonlocal operators. Discrete and Continuous Dynamical Systems- A, 35 (2015) no 12 6031-6068.

[27] J. NEčAs, Sur une méthode pour résoudre les équations aux dérivées partielles du type elliptique, voisine de la variationelle. Ann. Scuola Norm. Sup. Pisa Ser. 16 (1962) 305-326.

[28] I. Peral, F. Soria, Elliptic and Parabolic Equations involving the Hardy-Leray Potential. De Gruyter Series in Nonlinear Analysis and Applications. To appear 2020-21. 
[29] M. Riesz, Intégrales de Riemann-Liouville et potenciels. Acta Sci. Math. Szeged 9, (1938), 1-42.

[30] X. Ros-Отоn, J. Serra, The extremal solution for the fractional Laplacian. Calc. Var. Partial Differential Equations, 50, 2014, No 3-4, 723-750.

[31] L. Silvestre, Regularity of the Obstacle Problem for a Fractional Power of the Laplace Operator. Communications on Pure and Applied Mathematics, Vol. LX (2007), 67-112.

[32] E. M. Stein, G. Weiss, Fractional integrals on n-dimensional Euclidean space. J. Math. Mech., 7 (1958), 503-514.

[33] D. Yafaev, Sharp constants in the Hardy-Rellich inequalities. J. Functional Analysis, 168 (1999), no. 1, $121-144$.

* Laboratoire d’Analyse Nonlinéaire et Mathématiques Appliquées.

Département de Mathématiques, Université Abou Bakr Belkaïd, Tlemcen,

Tlemcen 13000, Algeria.

Departamento de Matemáticas,

Universidad Autónoma de Madrid, 28049, MAdRID, SPAin.

E-mail addresses: boumediene.abdellaoui@uam.es, ireneo.peral@uam.es, ana.primo@uam.es, 